\title{
ON THE LOSS OF GASES FROM A PLANETARY ATMOSPHERE
}

\author{
P. B. HAYS* and V. C. LIU \\ Department of Aeronautical and Astronautical Engineering \\ The University of Michigan, Ann Arbor
}

(Received 13 July 1965)

\begin{abstract}
An analysis of the collisional transition between the lower atmosphere and the collisionless exosphere is carried out based upon an integral formulation of the Boltzmann equation. This investigation utilizes a collision model which is a combination of Lorentz-gas and relaxation collision models. The results of this analysis indicate that intermolecular collisions act in two ways to affect the atmosphere. First, there is a critical layer, similar to the apparent photospheric surface of the Sun, from which the material escaping from the planet originates. This layer is determined by collision suffered by particles moving on orbits which skim tangentially by the planet. Secondly, collisions reduce the vertical flux of material in a manner analogous to the diffusion processes which occur in the lower atmosphere.
\end{abstract}

\section{INTRODUCTION}

A planetary atmosphere is naturally stratified into two broad regions: a lower continuum stratum within which intermolecular collisions prevail, and an upper stratum in which the atmospheric gases behave as a classical Knudsen gas. This differentiation results from the decrease of the collision frequency with height above the planetary surface. In the upper stratum particles moving with speeds in excess of the escape velocity can escape from the planetary gravitational field. Since material is escaping from the atmosphere and, conversely, being gained from the surrounding ambium, the state of a planetary atmosphere is obviously time-dependent. These facts were apparent to Waterston ${ }^{(1,2)}$ in 1846 when he presented his controversial paper on the kinetic theory of gases. Since that time, numerous investigations have been carried out in an attempt to clarify the nature of the escape process and to determine quantitatively the rate at which material is being lost. ${ }^{(1-17)}$

Many of those early investigations, ${ }^{(1-11,14)}$ and recent studies of the exosphere as well, ${ }^{(18-28)}$ are based on the concept of a critical level above which the atmosphere is considered collisionless and below which the atmosphere is treated as isothermal and collisiondominated. Under these circumstances the distribution function in the lower atmosphere takes the form given by Jeans ${ }^{(14)}$

$$
f_{i}^{*}\left(\mathrm{r}^{*}, \mathrm{~V}^{*}\right)=N_{i}^{*}\left(\beta_{i}^{*} / \pi\right)^{3 / 2} \exp \left[-\beta_{i}^{*}\left(V^{* 2}+V_{e}^{* 2}\left(r^{*}-R^{*}\right) / r^{*} R^{*}\right)\right]
$$

where $N_{i}^{*}$ is the number density of the $i$ th species at $r^{*}=R^{*}, \beta_{i}^{*}=m_{i}^{*} / 2 k T^{*}$ the inverse of the most probable particle speed squared, $V_{e}^{*}$ is the escape speed at $r^{*}=R^{*}$, and $R^{*}$ is a reference distance from the center of the planet. Here the star is used to indicate dimensional quantities.

If a critical level, $r_{\mathrm{cr}}{ }^{*}$, is assumed above which the collision frequency is zero and below which the atmosphere is collision-dominated, one finds that the distribution function given by Jeans ${ }^{(14)}$ implies an escape flux

$$
\mathscr{F} *=2 r_{\mathrm{cr}}{ }^{* 2} N_{i}^{*}\left(\pi / \beta_{i}{ }^{*}\right)^{1 / 2}\left(1+\beta_{i}{ }^{*} V_{e}^{* 2} R^{*} / r^{*}\right) \exp \left(-\beta_{i}^{*} V_{a}^{* 2}\right)
$$

a result obviously dependent upon the height of the critical level. This indeterminacy has

* On leave at Department of Applied Mathematics, Queen's University of Belfast, 1965-1966. 
been alleviated in many ways, but the most frequent assumption employed is to choose as the critical level the point at which a particle moving upward has a probability of $1 / e$ of escape without suffering a collision. (12)

Although the critical level model of the exosphere, which assumes a finite discontinuity in the collision frequency, offers a simple, convenient scheme for estimating the escape flux, it obviously does not represent the actual transition to the exosphere in which the collision frequency must be a continuous function of altitude. The inconsistencies of the critical level model were recognized by Milne ${ }^{(12)}$ and Jones, ${ }^{(13)}$ who improved the model by introducing a variable critical level which depended upon the zenith angle of a particle trajectory.

Recently Byutner ${ }^{(16,17)}$ further improved the model by considering in an approximate fashion the effect of distortions to the distribution function in the transition region. However, neither Byutner, Jones, nor Milne has attempted a self-consistent analysis based upon the kinetic theory; rather these theories, as does all previous work, rely upon a priori assumptions about the collisional processes which must determine the distribution function and consequently the escape flux within a transition region between the collision-dominated lower atmosphere and the collisionless exosphere.

To achieve a rational analysis of the escape flux and the transition to a collisionless exosphere, the present study proceeds from a set of self-consistent integral equations, deduced from the Boltzmann equation, governing the kinetic processes of the neutral particles situated near the lower boundary of the exosphere. On the basis of these equations the escape fluxes of separate major and minor constituent gases are evaluated.

\section{INTEGRAL EQUATIONS}

Consider a spherically symmetric atmosphere of neutral particles surrounding a planet. The velocity distribution function, $f_{i}^{*}$, is assumed to be a sufficient representation of the microscopic state of the gas. The function $f_{i}^{*}\left(\mathbf{r}^{*}, \mathbf{V}^{*}\right) d \mathbf{r}^{*} d \mathbf{V}^{*}$ represents the number of molecules of species $i$ in the element of volume $d \mathbf{r}^{*} d \mathrm{~V}^{*}$ surrounding the point $\mathbf{r}^{*}, \mathrm{~V}^{*}$ in the phase space, or $\mu$-space. Further, it is assumed that molecules experience only binary collisions and that the external forces acting upon a particle are conservative. Under these circumstances the distribution function for the $i$ th species of gas must satisfy the Boltzmann equation

$$
\begin{aligned}
\mathbf{V}^{*} \cdot \frac{\partial f_{i}^{*}}{\partial \mathbf{r}^{*}}-\frac{1}{m_{i}{ }^{*}} \frac{\partial \varphi^{*}}{\partial \mathbf{r}^{*}} \cdot \frac{\partial f_{i}^{*}}{\partial \mathbf{V}^{*}}=\sum_{j} \iint\left[f_{i}^{*}\left(\mathbf{V}_{i}{ }^{\prime \prime}\right) f_{j}{ }^{*}\left(\mathbf{V}_{j}{ }^{\prime \prime}\right)\right. & \left.-f_{i}^{*}\left(\mathbf{V}_{i}{ }^{*}\right) f_{j}\left(\mathbf{V}_{j}^{*}\right)\right] \\
& \times g^{*} I^{*}(g, x) d \Omega d \mathbf{V}_{i}{ }^{*}
\end{aligned}
$$

where the summation of $j$ includes all possible collisions suffered by the $i$ th species. Here $\mathrm{V}^{*}$ is the velocity of a particle, $m_{i}{ }^{*}$ the molecular mass of the $i$ th species, $\varphi^{*}$ the force potential, $g^{*}$ the relative velocity between colliding particles, $\left|\mathbf{V}_{i}^{*}-\mathbf{V}_{j}^{*}\right|, \chi$ is the angle of deviation of the relative velocity vector caused by a collision, $I$ the differential collision cross section and the prime ( )' denotes conditions after a collision. The reader is referred to reference ${ }^{(29)}$ for an extensive discussion of the Boltzmann equation for neutral particles.

The boundary conditions applicable to the non-ionized gases in the exosphere can be specified as follows:
(a) at $r^{*}=R^{*}$
$f_{i}^{*}\left(\mathbf{r}^{*}, \mathbf{V}^{*}\right)=F_{i}^{*}(|\mathbf{V}|)$
$\mu \geq 0$
(b) as $\mathbf{r}^{*} \rightarrow \infty$
$f_{i}^{*}\left(\mathbf{r}^{*}, \mathrm{~V}^{*}\right) \rightarrow 0$
$\mu<0$ 
where $\mu=\mathrm{r}^{*} \cdot \mathrm{V}^{*} /\left(\left|r^{*}\right|\left|V^{*}\right|\right)$. The first condition states that at some level $R^{*}$ the distribution function is known. This is realized at low levels where the collision frequency is high and consequently the distribution function is very nearly Maxwellian. The distribution function at this base level can be taken to be the local Maxwellian function

$$
F_{i}{ }^{*}\left(\left|V^{*}\right|\right)=N_{i}{ }^{*}\left(\beta_{i}{ }^{*} / \pi\right)^{3 / 2} \exp \left(-\beta_{i}{ }^{*} V^{* 2}\right)
$$

The second condition of (4) is somewhat more restrictive, because it implies that the planet is completely isolated so that no material is injected into the exosphere from the surrounding ambium. Although this is not generally the situation, one can neglect the crosscoupling between incoming particles and escaping particles as a first approximation. The only additional information required is a knowledge of the potential force acting on the particles. For a spherically symmetric planet, the Newtonian gravitational potential is given by the relation

$$
p^{*}\left(\mathbf{r}^{*}\right)=-G^{*} M_{0}^{*} m_{i}^{*} /\left|\mathbf{r}^{*}\right|
$$

where $G^{*}$ is the universal gravitational constant, and $M_{0}{ }^{*}$ is the mass of the planet and atmosphere below the level $r^{*}=R^{*}$. In this formulation the mass of material comprising the exosphere is assumed to have no significant effect upon the gravitational potential.

Before developing the formal solution to equation (3) for the distribution function it is convenient to decompose the collision term as follows:

and

$$
\sigma_{i}^{*}=\sum_{j} \iint f_{i}^{*}\left(\mathbf{V}_{i}^{* \prime}\right) f_{j}^{*}\left(\mathbf{V}_{j}^{* \prime}\right) g^{*} I^{*}\left(g^{*}, \chi\right) d \Omega d \mathbf{V}_{j}^{*}
$$

$$
v_{i}^{*}=\sum_{j} \iint f_{j}\left(\mathbf{V}_{j}^{*}\right) g^{*} I^{*}\left(g^{*}, \chi\right) d \Omega d \mathbf{V}_{j}^{*}
$$

These terms may be treated separately so long as one adheres to the concept that a collision is an isolated event. For this to be true, the effective range of intermolecular interaction must be much smaller than the mean distance between particles. This concept, although implicit in the binary collision assumption, is not rigorously satisfied by most molecular models. This condition is of minor importance if the collision term is not separated. ${ }^{(30)}$ However, when correctly defined, the quantity $v_{i}{ }^{*}$ is the collision frequency for a given particle with all encountering particles. The first integral, $\sigma_{i}{ }^{*}$, represents the net number of particles "produced" per second per unit volume of phase space due to collisions. Actually this process is not a production but a transfer of particles from one section of phase space to another due to the collisional process. These two quantities have physically meaningful definitions, and the collision frequency in particular is a most important parameter in transitional problems. In such problems it is especially useful to separate these terms rather than to mask their individual effects in combination. This is clear if one considers that the combined collision term is zero for both the collision-dominated and the collisionless cases, while in these extremes the collision frequency must vary by orders of magnitude.

In order to simplify the succeeding analysis, the following non-dimensional parameters will be introduced

$$
\begin{aligned}
& \mathrm{V}=\mathrm{V}^{*} / V_{0}^{*} \quad \sigma_{i}=\sigma_{i}^{*} R^{*} V_{i}^{* 2} / N_{i}^{*} \quad V_{i}^{*}=\left(2 G^{*} M_{0}^{*} / R^{*}\right)^{1 / 2} \\
& \mathrm{r}=\mathrm{r}^{*} / R^{*} \quad \nu_{i}=v_{i}{ }^{*} R^{*} / V_{e}^{*} \quad \mathscr{M}_{i}=\sqrt{ } \beta_{i}{ }^{*} V_{e}{ }^{*} \\
& f_{i}=f_{i}^{*} V_{e}^{* 3} / N_{i}^{*} \quad \varphi=\varphi^{*} / V_{e}^{* 2} \quad \mu=\mathbf{r}^{*} \cdot \mathbf{V}^{*} /\left|\mathbf{r}^{*}\right|\left|V^{*}\right|
\end{aligned}
$$


In terms of these coordinates and parameters the basic formulation of the problem becomes

$$
\mu V \frac{\partial f_{i}}{\partial r}-\frac{\mu}{2 r^{2}} \frac{\partial f_{i}}{\partial V}+\left[V / r-\frac{1}{2 V r^{2}}\right]\left(1-\mu^{2}\right) \frac{\partial f_{i}}{\partial \mu}=\sigma_{i}-v_{i} f_{i}
$$

where the distribution function $f_{i}$, is subject to the boundary conditions

$$
\begin{array}{lll}
\text { (a) at } r=1 & f_{i}(\mathbf{r}, \mathbf{V})=F_{i}(V) & \mu \geq 0 \\
\text { (b) as } r \rightarrow \infty & f_{i}(\mathbf{r}, \mathbf{V}) \rightarrow 0 & \mu<0
\end{array}
$$

Equation (10) can be treated formally by the method of characteristics. ${ }^{(3)}$ The characteristic equations in this case are:

$$
\frac{d r}{V \mu}=\frac{-2 r^{2} d V}{\mu}=\frac{d \mu}{\left[V / \mu-1 / 2 V r^{2}\right]\left(1-\mu^{2}\right)}=\frac{d f_{i}}{\left(\sigma_{i}-v_{i} f_{i}\right)}
$$

Equations (12) admit the following three independent integrals

$$
\begin{aligned}
E & =\frac{1}{2}\left(V^{2}-1 / r\right) \\
J^{2} & =r^{2} V^{2}\left(1-\mu^{2}\right) \\
f_{i}\left(\mathbf{r}_{1}, \mathbf{V}_{1}\right) & =f_{i}(\mathbf{r}, \mathbf{V}) \exp \left[+\int_{r_{1}}^{r}\left(\frac{y_{i}}{V \mu}\right) d \rho\right]-\int_{r_{1}}^{r}\left(\frac{\sigma_{2}}{V \mu}\right) \exp \left[+\int_{r_{1}}^{r}\left(\frac{y_{i}}{V \mu}\right) d \xi\right] d \rho
\end{aligned}
$$

where the integrations are carried out along paths of constant $E$ and $J$ in the phase space. It should be noted here that $E$ is the non-dimensional total energy of a particle and $J$ its non-dimensional angular momentum. In order to simplify the analysis $E$ and $J$ will be introduced as primary variables replacing $V$ and $\mu$. Note that in the inverse transformations

$$
\begin{aligned}
& V=(2 E+1 / r)^{1 / 2} \\
& \mu= \pm\left[1-J^{2} /\left(r^{2}(2 E+1 / r)\right)\right]^{1 / 2}
\end{aligned}
$$

$\mu$ is not uniquely defined in terms of the new coordinates. This difficulty is circumvented by splitting the distribution function such that

where

$$
f_{i}=f_{i}^{+}+f_{i}^{-}
$$

$$
f_{i}=f_{i}^{+} \text {for } \mu>0 \text { and } f_{i}=f_{i}^{-} \text {for } \mu<0
$$

In terms of this designation of $f_{i}$, one may formally solve the last equation of (13) for $f_{i}$ as follows:

and

$$
\begin{aligned}
f_{i}^{ \pm}\left(r, E, J^{2}\right)= & f_{i}^{( \pm)}\left(r_{1}, E, J^{2}\right) \exp \left[-\int_{r_{1}}^{r} Q_{i}^{( \pm)} d \rho\right] \\
& +\int_{r_{1}}^{r} P_{i}^{( \pm)} \exp \left[-\int_{\rho}^{r} Q_{i}^{( \pm)} d \xi\right] d \rho \\
Q_{i}^{ \pm}\left(\rho, E, J^{2}\right)= & \frac{v_{i}\left[\rho,(2 E+1 / \rho)^{1 / 2}, \pm\left(1-J^{2} / \rho^{2}(2 E+1 / \rho)\right)^{1 / 2}\right]}{\left(2 E+1 / \rho-J^{2} / \rho^{2}\right)^{1 / 2}}
\end{aligned}
$$

where

$$
P_{i}^{ \pm}\left(\rho, E, J^{2}\right)=\frac{\sigma_{i}\left[\rho,(2 E+1 / \rho)^{1 / 2}, \pm\left(1-J^{2} / \rho^{2}(2 E+1 / \rho)\right)^{1 / 2}\right]}{\left(2 E+1 / \rho-J^{2} / \rho^{2}\right)^{1 / 2}}
$$

$$
\sigma_{i}=\sigma_{i}(r, V, \mu) \text { and } \nu_{i}=\nu_{i}(r, V, \mu)
$$


This form for $f_{i}$ is not complete because the integrals in equation (16) are not completely specified. The integration is to be carried out along paths of constant $E$ and $J^{2}$. These paths are easily recognized as being the paths that a particle would follow if there were no collisions. That is, the two integrals $E$ and $J^{2}$ are the energy and angular momentum of a particle with velocity $V$ and direction $\mu$; thus a path of constant $E, J^{2}$ is identical to the path followed by a point mass moving in the planetary gravitational field. The quantity $E$ is the total energy, which is negative for trapped particles. One important property of the motion of a particle in an attracting central force field is the existence of bound orbits, ${ }^{(32)}$ or closed characteristics. The extreme values of $r$ at which the closed characteristics have minima or maxima are the points at which $\mu=0$. At these points the direction of integration in equation (16) must be changed, and $Q^{ \pm}$and $P^{ \pm}$change to the corresponding $Q^{\mp}$ and $P^{\mp}$, depending upon the sign of $\mu$ after the change in direction; hence the $( \pm)$ in equation (16).

A second difficulty results from the necessity to know at which boundary, if any, the characteristic originates, so that bounding values of $f_{i}\left(\mathbf{r}_{1}, \mathbf{V}\right)$ may be determined. However once the extreme values of $r$ are known for a given characteristic it is an elementary task to determine which characteristics originate at the planetary surface and which originate at infinity. The only remaining problem is to determine the limiting values of $r$ on a given characteristic where $E$ and $J^{2}$ are constant. This is easily accomplished by setting $\mu=0$ in equation (14) and solving for the values of $r$.

1. For $E>0$

2. For $E=0$

$$
r=r_{p}=-(1 / 4 E)\left(1-\sqrt{ }\left(1+8 E J^{2}\right)\right)
$$

3. For $E<0$

$$
r=r_{p}=J^{2}
$$

$$
\begin{aligned}
& r=r_{a}=-(1 / 4 E)\left(1+\sqrt{ }\left(1+8 E J^{2}\right)\right) \\
& r=r_{p}=-(1 / 4 E)\left(1-\sqrt{ }\left(1+8 E J^{2}\right)\right)
\end{aligned}
$$

where $r_{a}$ is the maximum, sometimes referred to as apogee distance, and $r_{p}$ is the minimum, called the perigee distance. Notice that cases 1 and 2 represent unbounded characteristics which have a single minimum and no maximum. Case 3 represents completely bounded paths with $r_{p} \leq r \leq r_{a}$. When the path penetrates the sphere $r=1, r_{p}<1$, or in terms of $E, J^{2}$

$$
E \geq J^{2} / 2-\frac{1}{2}
$$

Furthermore, since $\mu$ is a real quantity

$$
E>J^{2} / 2 r^{2}-\frac{1}{2} r
$$

This information is summarized in Fig. 1, with the labels I and II referring to open and closed characteristics respectively, the subscripts $a$ and $b$ referring to cases having minimum radius $r_{p}<1$ and $r_{p}>1$ respectively. The shaded region represents characteristics which originate at the planetary surface. Figure 2 is a schematic diagram of these various characteristic paths.

It is interesting to note that in regions $\mathrm{I}_{b}{ }^{+}$and $\mathrm{I}_{b}-$ the characteristic paths are bounded and reach neither of the boundaries. In the past, the study of these bound or satellite orbits has been made difficult by the lack of a meaningful boundary condition. However the above-mentioned difficulty does not present itself when a collisional analysis such as 


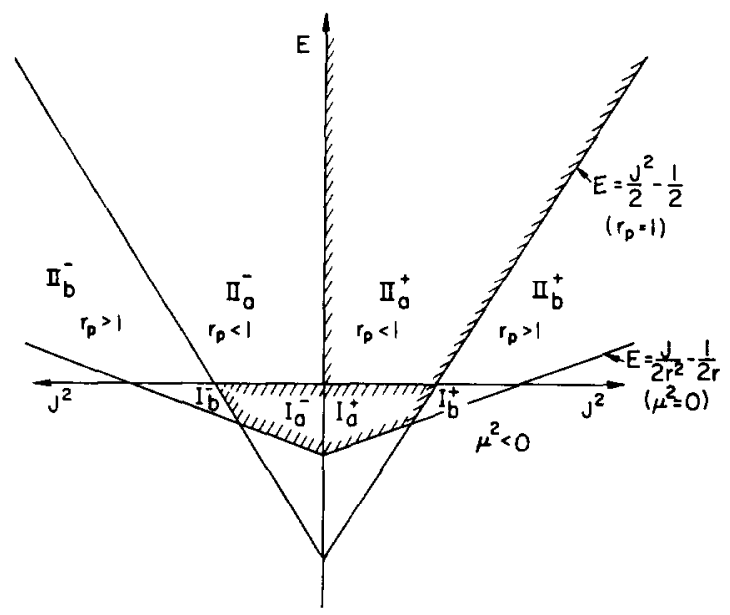

Fig. 1. Characteristic limits in the $E, J^{2}$ Plane.

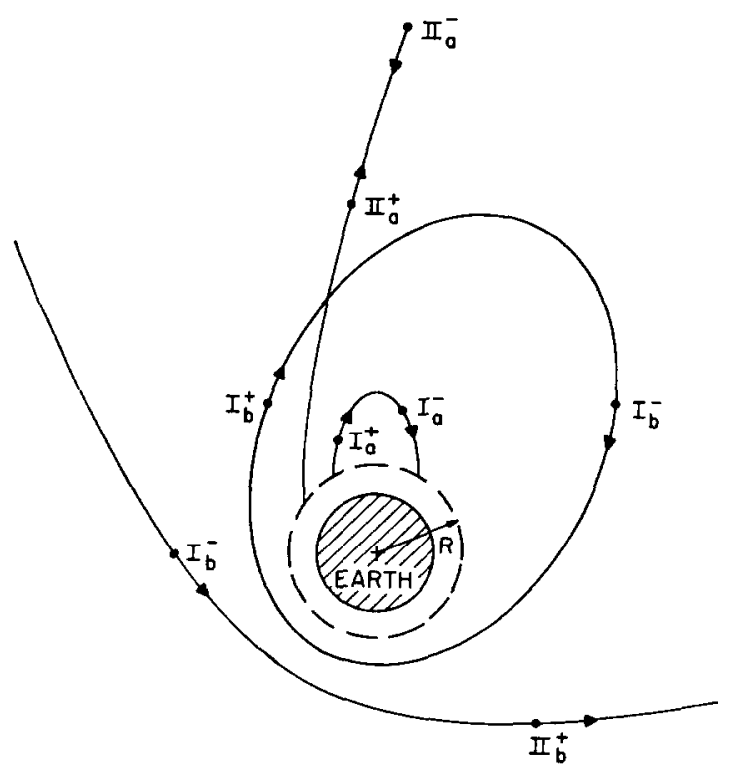

Fig. 2. TYPiCAL CHARACTERISTIC PATHS, WHICH ARE IDENTICAL to tHE TRAJECTORY OF A PARTICLE IN A VACUUM.

the present study is used. Under these circumstances $r_{1}$ may be set equal to $r$ and the integration carried out around the entire path. The result is a compatibility condition which determines $f_{i}$. This relation for $f_{i}$ may be obtained from equation (16) by setting $r_{1}=r$ and solving for $f_{i}$ as follows:

$$
f_{i}\left(r, E, J^{2}\right)=\frac{\oint P_{i}{ }^{( \pm)} \exp \left(-\oint_{\rho}^{r} Q_{i}^{( \pm)} d \xi\right) d \rho}{1-\exp \left(-\oint Q_{i}{ }^{ \pm} d \xi\right)}
$$

The equation for $f_{i}^{ \pm}$may now be written specifically for the various regions. 
1. In region $\mathrm{I}_{a}^{ \pm}$

and

$$
f_{i}^{+}\left(r, E, J^{2}\right)=F_{i}(E) \exp \left(-\int_{1}^{r} Q_{i}+d \xi\right)+\int_{2}^{r} P_{i}+\exp \left(-\int_{\rho}^{r} Q_{i}+d \xi\right) d \rho
$$

$$
f_{i}^{-}\left(r, E, J^{2}\right)=f_{i}^{+}\left(r_{a}, E, J^{2}\right) \exp \left(-\int_{r}^{r_{a}} Q_{i} d \xi\right)+\int_{r}^{r_{a}} P_{i}-\exp \left(-\int_{r}^{\rho} Q_{i}-d \xi\right) d \rho
$$

This region is commonly referred to as the ballistic component.

2. In region $\mathrm{I}_{b}^{ \pm}$

$$
\begin{gathered}
f_{i}^{ \pm}\left(r, E, J^{2}\right)=\exp \left(\mp \int_{r_{p}}^{r} Q_{i}^{ \pm} d \xi\right)\left\{ \pm \int_{r_{p}}^{r} P_{i}^{ \pm} \exp \left( \pm \int_{r_{p}}^{\rho} Q_{i}^{ \pm} d \xi\right) d \rho\right. \\
+\frac{1}{\sinh \left[\int_{r_{p}}^{r_{a}}\left(\frac{Q^{+}+Q^{-}}{2}\right) d \xi\right]}\left(\int_{r_{p}}^{r_{a}} P_{i}^{+} \exp \left[+\int_{r_{p}}^{\rho} Q^{+} d \xi-\int_{r_{p}}^{r_{a}}\left(\frac{Q^{+}+Q^{-}}{2}\right) d \xi\right]\right. \\
\left.\left.+P_{i}^{-} \exp \left[-\int_{r_{p}}^{\rho} Q^{-} d \xi+\int_{r_{p}}^{r_{a}}\left(\frac{Q^{+}+Q^{-}}{2}\right) d \xi\right] d \rho\right)\right\}
\end{gathered}
$$

This region is the satellite component, which has no definite boundary condition.

3. In region $\mathrm{II}_{a} \pm$

and

$$
f_{i}^{+}\left(r, E, J^{2}\right)=F_{i}(E) \exp \left(-\int_{1}^{r} Q_{i}^{+} d \rho\right)+\int_{1}^{r} P_{i}^{+} \exp \left(-\int_{\rho}^{r} Q_{i}^{+} d \xi\right) d \rho
$$

$$
f_{i}^{-}\left(r, E, J^{2}\right)=\int_{r}^{\infty} P_{i}-\exp \left(-\int_{r}^{\rho} Q_{i}^{-} d \xi\right) d \rho
$$

This region is the ballistic component, which can escape from the planet, and $f_{i}^{-}$is the return flux determined entirely by collisions.

4. In region $\mathrm{II}_{b}^{ \pm}$

and

$$
f_{i}^{-}\left(r, E, J^{2}\right)=\int_{r}^{\infty} P_{i}^{-} \exp \left(-\int_{r}^{0} Q_{i}^{-} d \xi\right) d \rho
$$

$$
f_{i}^{+}\left(r, E, J^{2}\right)=f_{i}^{-}\left(r_{p}, E, J^{2}\right) \exp \left(-\int_{r_{p}}^{r} Q_{i}+d \xi\right)+\int_{r_{p}}^{r} P_{i}^{+} \exp \left(-\int_{\rho}^{r} Q_{i}+d \xi\right) d \rho
$$

This component is determined entirely by collisions and is absent in the collisionless solution.

The physical significance of these integral relations for $f_{i}$ can readily be seen by rewriting the general relation (16) in terms of the distances along the characteristics

$$
f_{i}\left(s, E, J^{2}\right)=f_{i}\left(O, E, J^{2}\right) \exp \left(-\int_{0}^{s} d s / l(s)\right)+\int_{0}^{s}\left[\sigma_{i}(s) / V(s)\right] \exp \left(-\int_{s^{\prime}}^{s} d s^{\prime \prime} / l(s)\right) d s^{\prime}
$$

where $s$ is the path length measured from the boundary at $r=r_{1}$, and where $l(s)=V(s) / v_{i}(s)$ is the free path between collisions in an isotropic medium with collision frequency $v_{i}(s)$ for a particle moving with velocity $V(s)$. In equation (32) the quantity $\exp \left(-\int_{0}^{8} \frac{d s^{\prime}}{l}\right)$ is the 
probability that a particle will travel the distance $s$ without suffering a collision. ${ }^{(13)}$ Thus, the first term on the right-hand side of equation (32) accounts for the number of particles on the characteristic at $s^{\prime}=0$ which ultimately reach $s$. The second term accounts for the number of particles which, due to collisions, are deflected onto the characteristic and ultimately reach $s$. Here, the effects of chemical reactions and ionization are assumed to be small compared with molecular collisions.

\section{LIMITING SOLUTIONS}

The integral equations developed in the preceding section admit two limiting solutions corresponding to the completely collision-dominated situation and its collisionless counterpart. It is useful to develop these limiting situations before proceeding to the more complex cases.

\section{(a) Collision-dominated solution}

When the collision frequency becomes extremely large or the mean free path sufficiently small one can write equation (32) in the following form:

$$
f_{i}\left(s, E, J^{2}\right)=F_{i}(E) \exp \left(-\int_{0}^{s} d s^{\prime} / l\right)+\int_{0}^{s}\left(\sigma_{i} / v_{i}\right) \frac{d}{d s^{\prime}} \exp \left(-\int_{z^{\prime}}^{s} d s^{\prime \prime} / l\right) d s^{\prime}
$$

thus

$$
\begin{gathered}
f_{i} \rightarrow \sigma_{i}(s) / v_{i}(s) \quad \text { as } \quad l(s) \rightarrow 0 \\
\sigma_{i}-v_{i} f_{i}=0
\end{gathered}
$$

But this is the condition for the distribution to be Maxwellian. ${ }^{(14)}$ The solution in this situation is

$$
f_{i}\left(r, E, J^{2}\right)=F_{i}(E)=\left(\mathscr{H}_{i}^{3} / \pi^{3 / 2}\right) \exp \left[-\mathscr{M}_{i}^{2}(2 E+1)\right]
$$

or in terms of the conventional velocity coordinates

$$
f_{i}(\mathbf{r}, \mathbf{V})=\left(\mathscr{M}_{i}{ }^{3} / \pi^{3 / 2}\right) \exp \left[-\mathscr{M}_{i}{ }^{2}\left(V^{2}+1-1 / r\right)\right]
$$

This, of course, is the limiting case where transport fluxes vanish. In this situation the number density has the value

$$
n_{\imath}(r)=\exp \left[-\mathscr{M}_{i}^{2}(1-1 / r)\right]
$$

which identifies with the density of the isothermal atmosphere ubtained from the hydrostatic equations. This solution represents the lower atmosphere where the collisions are frequent. The presence of transport fluxes and radiation processes tend to deviate the molecular distribution from the ideal locally Maxwellian state.

\section{(b) Collisionless solution}

In the other extreme, where the collision frequency is zero above the boundary level, one obtains the collisionless solution as given by Aamodt and Case. ${ }^{(27)}$ When $\sigma_{i}=v_{i}=0$ equations (25)-(30) reducc to

$$
f_{i}\left(r, E, J^{2}\right)=\left\{\begin{array}{l}
\mathrm{O} \text { in } \mathrm{II}_{a}^{-}, \mathrm{II}_{b}{ }^{ \pm} \\
F_{i}(E) \text { in } \mathrm{I}_{a}{ }^{ \pm}, \mathrm{II}_{a}{ }^{+} \\
\text {undefined in } \mathrm{I}_{b}{ }^{ \pm}
\end{array}\right.
$$


Here the solution was taken to be Maxwellian at $r=1$. This solution has commonly been taken as a satisfactory model for the exosphere and has been used extensively to determine the escape flux. The number density in the collisionless case can be evaluated from the distribution function (37) if an appropriate assumption is made concerning the undefined satellite components in regions $\mathrm{I}_{b}{ }^{ \pm}$. Here it is assumed that the satellite orbits are not populated in agreement with the assumptions of Aamodt and Case. ${ }^{(27)}$ Thus one finds

$$
\begin{aligned}
n_{i}= & (1 / 2) \exp \left[-\mathscr{M}_{i}{ }^{2}(1-1 / r)\right]\left\{1+\operatorname{erf}\left(\mathscr{M}_{i} / \sqrt{ } r\right)\right. \\
& -\sqrt{ }\left(1-1 / r^{2}\right)\left[1+\operatorname{erf}\left(\mathscr{M}_{2} / \sqrt{ }(r+1)\right)\right] \exp \left[-\mathscr{M}_{i}{ }^{2} / r(r+1)\right] \\
& \left.+\left(2 \mathscr{M}_{i} / \sqrt{ }(\pi r)\right)(\sqrt{ }(1-1 / r)-1) \exp \left[-\mathscr{M}_{i}{ }^{2} / r\right]\right\}
\end{aligned}
$$

where $\operatorname{erf}(x)=(2 / \sqrt{ } \pi) \int_{0}^{x} \exp \left(-t^{2}\right) d t$.

\section{(c) Comparison of limiting results}

The distribution functions for the two limiting solutions are closely related since the collisionless solution is equal to the collision-dominated solution except for particles which have escaped and those on the satellite orbits, for which the distribution function is assumed to be zero. Near the base level the differences between these limits are small when one considers the macroscopic properties of the atmosphere. For instance, the number densities of the two solutions differ as follows near the base level

$$
\frac{n_{\text {C.D. }}-n_{\text {C.L }}}{n_{\text {C.D }}}=\frac{\Delta n}{n_{i}} \cong\left(2 \mathscr{H}_{i} / \sqrt{ }(\pi r)\right) \exp \left(-\mathscr{H}_{i}^{2} / r\right)
$$

for $\mathscr{M}_{i} \gg 1$ and $r \cong 1$. These variations grow as the altitude above the boundary level is increased until the two solutions exhibit widely varying number densities as $r \rightarrow \infty$. The collisionless solution tends to zero as $r \rightarrow \infty$, while the collision-dominated solution tends to a constant number density. This same behaviour is exhibited by the other macroscopic properties of the atmosphere except for the escape flux, which is zero for the collisiondominated solution. The loss of material in the collisionless case can be expressed in terms of the escape flux

$$
\mathscr{F}_{i}=4 \pi r^{2} \overline{n(r) u_{r}(r)}=2 \sqrt{\pi}\left(\mathscr{H}_{i}+1 / \mathscr{M}_{i}\right) \exp \left(-\mathscr{M}_{i}^{2}\right)
$$

For reference, this flux is presented in Fig. 3. The collision-dominated solution presented here has excluded the possibility of transport fluxes, however this is not physically realistic, and indeed Bates ${ }^{(33)}$ and Bates and Patterson ${ }^{(34,35)}$ have shown that for light-weight gases such as hydrogen the upward flux of material caused by escape has a profound effect upon the number density of these constituents within the lower atmosphere. The number density may be reduced by an order of magnitude for hydrogen in the Earth's atmosphere due to material being removed more rapidly by escape than it can be replenished by diffusion from below.

Thus, although these two limiting solutions appear similar in terms of macroscopic properties near the transition level, it is to be expected that the coupling between the lower and upper atmosphere in the transition region will profoundly affect both the thermosphere and exosphere. What is needed is a model which spans the collisional transition region and which includes a detailed examination of the collision processes. 


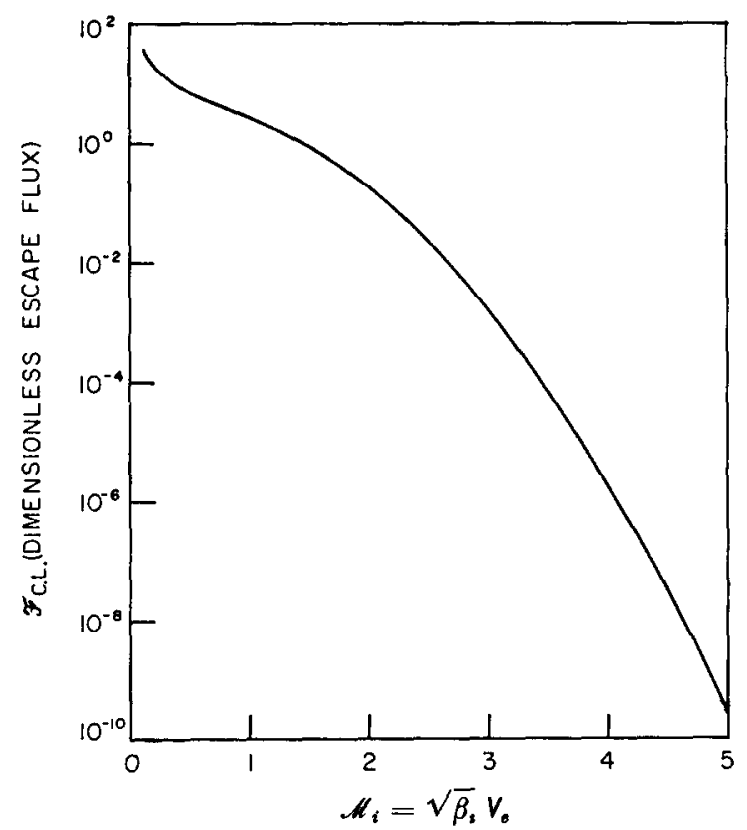

Fig. 3. EsCAPE fluX PRedicted hy THE COLLISIONLESS theORY.

\section{ESCAPE OF A MINOR CONSTITUENT GAS}

It has been suggested that the assumption of quiescence and the neglect of the collisional transition between the lower and upper atmosphere have serious consequences on the present theories of atmospheric escape. In order to pursue this question, the escape of a minor constituent gas will be considered in detail. The formalism necessary for this examination was developed above, however a molecular collision model must be postulated in order to proceed.

\section{(a) The relaxed Lorentz-gas collision model}

In the past, three general techniques have been used to simulate mathematically the effects of collisions. First, the relatively exact formulation of the binary collision integrals combined with an intermolccular force law can be used to relate the collisional production and loss to the distribution function. This is of course the preferred choice, but the complexity of this approach has serious disadvantages in involved problems like that of atmospheric escape. The second approach is the simple choice of postulating the collisional effects a priori, neglecting entirely the coupling with the actual distribution function. Jones ${ }^{(13)}$ and Byutner ${ }^{(16)}$ used this method with limited success, and although their results are useful in a limited sense, the method cannot be used either to obtain the effects of upward motion, or to relate the changes in the collisional production to the changes in the distribution function resulting from the loss of material by escape. These effects must be considered for the minor constituent gases with low molecular weight. The third alternative is to use an approximate collision term, in the spirit of the Krook's ${ }^{(3 \theta)}$ relaxation model, which is a compromise between these two extremes. However, such a model must be chosen with extreme care in order to be as exact as possible without being so cumbersome as to make the analysis intractable. 
In developing a kinetic model appropriate for the study of the escape of a light-weight minor constituent gas we begin with a review of the properties of the classical Lorentz-gas. This hypothetical gas represents the' situation in which $n_{i} / n_{B} \ll 1$ and $m_{i} / m_{B} \rightarrow 0$, that is, where a minor gas is diffusing through a background of infinitely massive scatters. Under these circumstances a collision changes the direction in which a molecule is moving, but never changes its speed. This is illustrated by the collision integrals for hard spherical molecules satisfying the Lorentz-gas limits, where the collision frequency is

and the production is

$$
v_{i}=\pi\left(d_{i B}^{* 2} \eta_{B}^{*}(r) R^{*}\right) V_{i}
$$

$$
\sigma_{i}=\left(v_{i} / 2\right) \int_{-1}^{+1} f_{i}(r, E, \mu) d \mu
$$

where $d_{i B}{ }^{*}$ is the collision diameter and the subscript $B$ refers to the background gas. Here all directions of motion are equally probable after a collision, but the speed is unchanged. Thus if this kinetic model were employed in the escape problem the effects of collisions would be confined to that portion of the distribution function where molecules have speeds in excess of the escape velocity. However, in the real atmosphere, the mass ratio may be small but is always non-zero; thus collisions do change the speed of a particle and consequently, after sufficient time distortions to the distribution function, will spread to all regions of the velocity space.

A real gas of non-zero mass will exhibit two relaxation times: first, a rapid relaxation of the anisotrophies in the distribution function in the order of a single collision time; this rapid angular relaxation is followed by a gradual decay of the residual isotropic distortion to the local Maxwellian state in a time which depends upon the ratios of the mass of the relaxing gas to that of the background gas. This problem is important in the theory of weakly ionized gases, ${ }^{(37)}$ where the slow relaxation to the Maxwellian state can be realized by adding a correction term to the Lorentz-gas production. This correction term is similar to Krook's single relaxation time collision model ${ }^{(36)}$ used in ordinary rarefied gas dynamics. The addition of a smoothing influence, such as the relaxation model, causes the distribution of molecular velocities to approach the Maxwellian state as required, while still exhibiting the rapid smoothing of the Lorentz gas.

A similar model can be used to study the escape of a minor constituent gas of low molecular weight. That is, a relaxed Lorentz-gas molecular model can be postulated which has the following functional form:

and

$$
v_{i}=\pi\left(d_{i B}^{* 2} \eta_{B}^{*}(r) R^{*}\right) V_{i}
$$

$$
\sigma_{i}=\nu_{i} F_{i}(E)\left\{(1-\eta)(1 / 2) \int_{-1}^{+}\left[f_{i}(r, E, \mu) / F_{i}(E)\right] d \mu+\eta\right\}
$$

where $\eta$ is a parameter related to the isotropic relaxation of the minor gas. This form for the collision operator is a compromise between the Lorentz-gas model ${ }^{(37)}$ where $\eta=0$ and a purcly relaxation model ${ }^{(36)}$ where $\eta=1$. Notice that the Lorentz gas collision frequcncy $v_{i}$, has been retained since the escape velocity is usually very large compared to the thermal speed.

The physical meaning of the parameter $\eta$ and the logic behind this particular functional form for $\sigma_{i}$ can be understood from the following simple illustration. Consider the temporal change of the non-Maxwellian distribution function in a homogeneous gas which has the 
modified Lorentz-gas collision model postulated in equations (40) and (41). The distribution function depends only upon time and velocity, thus satisfying the unsteady Boltzmann equation

$$
\frac{\partial f_{i}}{\partial t}=\sigma_{i}-v_{i} f_{i}
$$

Introduce the function

$$
\mathscr{L}(E, t)=(1 / 2) \int_{-1}^{+1}\left[f_{i}(r, E, \mu) / F_{i}(E)\right] d \mu
$$

and integrate equation (42) over $\mu$ to obtain the moment equation

$$
\frac{\partial \mathscr{L}}{\partial t}=v_{i}[(1-\eta) \mathscr{L}+\eta-\mathscr{L}]
$$

This equation has the solution

$$
\mathscr{L}(E, t)=1+(\mathscr{L}(E, 0)-1) e^{-v_{\imath} \eta t}
$$

indicating that $\left(\nu_{i} \eta\right)^{-1}$ may be interpreted as the time constant for relaxation of the isotropic distortions of the distribution function of the local Maxwellian $F_{i}(E)$. Thus, $1 / \eta$ is a measure of the average number of collisions required for a distortion of the distribution function from Maxwellian to be reduced by a factor $1 / e$. Here, the relaxation rate is independent of the velocity of the particles being considered and $\eta$ is called the average relaxation rate parameter.

\section{(b) Average relaxation rate parameter (hard spherical molecules)}

The relaxed Lorentz-gas kinetic model is complete only when the relaxation rate parameter $\eta$ is known. This parameter forms the connection between the mathematical model proposed here and the gas mixture that the model is to simulate. However, at this point one is faced with a rather delicate decision: Because the average relaxation rate parameter has not been defined precisely, the definition of this parameter represents a critical step in bridging the gap between the theoretical model and the physical reality that this model is to represent. In order to resolve this indeterminacy, we decided to combine the exact definition of $\sigma_{i}$ from equation (7) with the approximation (41) and average over the region of velocity space of interest to determine $\eta$; that is, to equate the two equations and to average them such that

$$
\begin{array}{r}
(1-\eta)\left\langle\left(v_{\imath} / 2\right) \int_{-1}^{+1} f_{i}(r, E, \mu) d \mu\right\rangle+\eta\left\langle v_{i} F_{i}(E)\right\rangle \\
=\left\langle\iint f_{i}\left(\mathbf{V}_{i}{ }^{\prime}\right) F_{B}\left(\mathbf{V}_{B}{ }^{\prime}\right) g I(g, \chi) d \Omega d \mathbf{V}_{B}\right\rangle
\end{array}
$$

where the bracket \langle\rangle indicates the integral over the velocity space for all velocities larger than the escape speed. This definition for $\eta$ insures that the total number of particles produced by collisions with speed greater than the escape speed will be correctly represented by the approximate collision model proposed here. We further decided that for the present application a representative value of $\eta$ could be obtained by evaluating the collisional production operator for a specific distribution function which incorporates the main features associated 
with the exosphere. In particular, an equilibrium Maxwellian distribution truncated at the escape velocity is chosen

$$
f_{i}(r, E, \mu)=\left\{\begin{array}{cc}
F_{i}(E) & V \leq 1 \\
0 & V>1
\end{array}\right.
$$

Combining the distribution function with the definition for $\eta$ chosen above yields the relation

$$
\eta=\frac{\int_{1}^{\infty} \int_{0}^{2 \pi} \int_{0}^{\pi} \sigma_{i} V^{2} \sin \theta d \theta d \alpha d V}{\int_{1}^{\infty} \int_{0}^{2 \pi} \int_{0}^{\pi} v_{i} F_{i}(E) V^{2} \sin \theta d \theta d \alpha d V}
$$

where $\sigma_{i}$ is obtained by evaluating equation (7) where $f_{i}$ is defined by (45) and $f_{f}=F_{B}$ the equilibrium Maxwellian distribution function for the background gas. For a minor constituent gas composed of hard spherical molecules, equation (7) is linearly dependent upon $f_{i}$ and can be partially integrated to give the relation

$$
\sigma_{i}=\left[\left(d_{i B}{ }^{* 2} N_{B}{ }^{*}(R) R^{*}\right) \mathscr{M}_{B} F_{2}(E) / \sqrt{ } \pi\right] \iiint_{-\infty}^{+\infty} L_{i}\left(\mathbf{V}_{i}^{\prime}\right) \exp \left[-\mathscr{M}_{B}{ }^{2}\left(Q+V_{i} \cos \varphi\right)\right] Q^{-1} d \mathbf{Q}
$$

where

$$
\begin{aligned}
L_{i} & =f_{i} / F_{i}(E) \\
\mathbf{Q} & =\left(\left(m_{i}+m_{B}\right) / 2 m_{B}\right)\left(\mathbf{V}_{i}^{\prime}-\mathbf{V}_{i}\right) \\
\cos \varphi & =\mathbf{Q} \cdot \mathbf{V}_{i} /\left(|\mathbf{Q}|\left|\mathbf{V}_{i}\right|\right) .
\end{aligned}
$$

Equation (48) is derived in a manner similar to that used in section 7.6 of reference ${ }^{(29)}$ to develop the linear Boltzmann equation collision operator.

The collisional production, $\sigma_{i}$, can be evaluated for the limiting cases of mass ratio equal to 1 and mass ratio very small. The results for these two situations are:

(a) $m_{i} / m_{B}=1$

(1) $V_{i} \geq 1$

$$
\begin{gathered}
\sigma_{i}=\left(d_{i B}{ }^{* 2} N_{B}{ }^{*}(R) R^{*}\right)\left(\mathscr{M}_{i}{ }^{3} /\left(\mathscr{M}_{B}{ }^{2} V_{i}\right)\right) \exp \left(-\mathscr{M}_{i}{ }^{2} V_{i}^{2}\right) \\
\times\left\{\left(\mathscr{M}_{i}{ }^{2}-\frac{1}{2}\right) \operatorname{erf} \mathscr{M}_{i}+\left(\mathscr{H}_{i} / \sqrt{ } \pi\right) \exp \left(-\mathscr{M}_{i}{ }^{2}\right)\right\} \\
\begin{array}{c}
(2) V_{i} \leq 1 \\
\sigma_{i}=\left(d_{i B}{ }^{* 2} N_{B}{ }^{*}(R) R^{*}\right)\left(\mathscr{M}_{i}{ }^{3} /\left(\mathscr{M}_{B}{ }^{2} V_{1}\right)\right) \exp \left(-\mathscr{M}_{i}{ }^{2} V_{i}{ }^{2}\right) \\
\times\left\{\left[\mathscr{M}_{i}{ }^{2} V^{2}+\frac{1}{2}-\exp \left(-\mathscr{M}_{i}{ }^{2}\left(V_{i}{ }^{2}-1\right)\right)\right] \operatorname{erf}\left(\mathscr{M}_{i} V_{i}\right)\right. \\
\left.+\left(\mathscr{M}_{i} V_{i} / \sqrt{ } \pi\right) \exp \left(-\mathscr{M}_{i}{ }^{2} V_{i}{ }^{2}\right)\right\}
\end{array}
\end{gathered}
$$

(b) $m_{i} / m_{B} \ll 1$

(1) $V_{i} \geq 1$

$$
\begin{aligned}
\sigma_{i}= & \left(d_{i B}{ }^{* 2} N_{B}{ }^{*}(R) R^{*}\right)\left(\mathscr{H}_{i}{ }^{3} /\left(\mathscr{M}_{B}{ }^{2} V_{i}\right)\right) \exp \left(-\mathscr{M}_{i}{ }^{2} V_{i}{ }^{2}\right) \\
& \times\left\{\left[\mathscr{K}_{B}{ }^{4}\left(\left(1-V_{i}^{2}\right) / 4\right)^{2}+\mathscr{M}_{B}{ }^{2} V_{i}^{2} / 2+\frac{1}{4}\right] .\right. \\
& \times\left[\operatorname{erf}\left(\mathscr{M}_{B}\left(V_{i}+1\right) / 2\right)-\operatorname{erf}\left(\mathscr{M}_{B}\left(V_{i}-1\right) / 2\right)\right] \\
& +\left[\left(3 V_{i}-1\right) \mathscr{H}_{B} / 4+\mathscr{M}_{B}{ }^{3}\left(V_{i}-1\right)^{2}\left(V_{i}+1\right) / 8\right] \exp \left(-\mathscr{M}_{B}{ }^{2}\left(V_{i}+1\right)^{2} / 4\right) / \sqrt{ } \pi \\
& \left.-\left[\left(3 V_{i}+1\right) \mathscr{M}_{B} / 4+\mathscr{K}_{B}{ }^{3}\left(V_{i}-1\right)\left(V_{i}+1\right)^{2} / 8\right] \exp \left(-\mathscr{K}_{B}{ }^{2}\left(V_{i}-1\right)^{2} / 4\right) / \sqrt{ } \pi\right\}
\end{aligned}
$$


(2) $V_{i} \leq 1$

$$
\begin{aligned}
& \left.\sigma_{i}=d_{i B}{ }^{* 2} N_{B}^{*}(R) R^{*}\right)\left(\mathscr{M}_{i}^{3} /\left(\mathscr{M}_{B}^{2} V_{i}\right)\right) \exp \left(-\mathscr{M}_{i}^{2} V_{i}^{2}\right) \\
& \times\left\{\left(\mathscr{M}_{B}{ }^{2} V_{i}^{2}+\frac{1}{2}\right) \operatorname{erf}\left(\mathscr{H}_{B} V_{i}\right)+\left(\mathscr{H}_{B} V_{i} / \sqrt{ } \pi\right) \exp \left(-\mathscr{M}_{B}{ }^{2} V_{i}^{2}\right)\right. \\
& -\left[\mathscr{H}_{B}^{4}\left(1-V_{i}^{2}\right)^{2} / 16+\mathscr{H}_{B}^{2} V_{i}^{2} / 2+\frac{1}{4}\right]\left[\operatorname{erf}\left(\mathscr{M}_{B}\left(V_{i}+1\right) / 2\right)\right. \\
& \left.+\operatorname{erf}\left(\mathscr{M}_{B}\left(V_{i}-1\right) / 2\right)\right] \\
& -\left[\left(3 V_{i}+1\right) \mathscr{M}_{B} / 4+\mathscr{M}_{B}{ }^{3}\left(V_{i}-1\right)\left(V_{i}+1\right)^{2} / 8\right] \exp \left(-\mathscr{M}_{B}{ }^{2}\left(V_{i}-1\right)^{2} / 4\right) / \sqrt{ } \pi \\
& \left.-\left[\left(3 V_{i}-1\right) \mathscr{M}_{B} / 4+\mathscr{H}_{B}^{3}\left(V_{i}-1\right)^{2}\left(V_{i}+1\right) / 8\right] \exp \left(-\mathscr{H}_{B}{ }^{2}\left(V_{i}+1\right)^{2} / 4\right) / \sqrt{ } \pi\right\}
\end{aligned}
$$

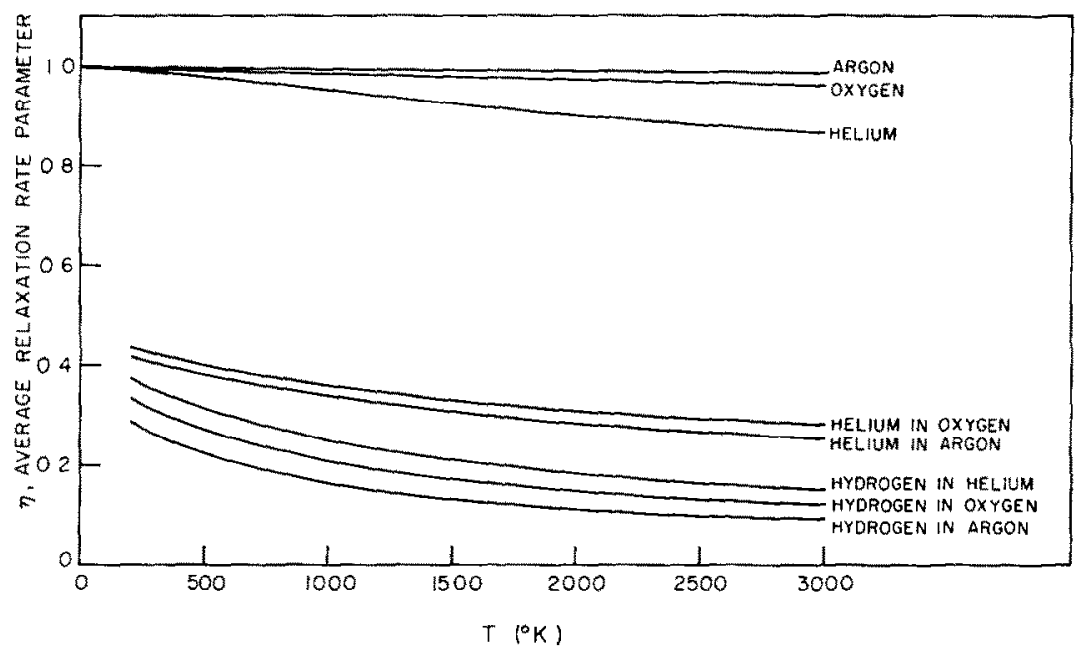

Fig. 4. RELAXATION RATE PARAMETER AS A FUNCTION OF TEMPERATURE FOR VARIOUS GAS MIXTURES.

The average relaxation rate parameter can be obtained directly from these relations using the definition provided in equation (47). For the first case where $m_{\imath} / m_{B}$ equals 1 , that is for a minor gas of the same molecular weight as the background gas, the relaxation rate parameter can be obtained by direct integration:

(a) $m_{i} / m_{B}=1$

$$
\begin{aligned}
\eta= & \left\{\left(\mathscr{M}_{i}^{2}-\frac{1}{2}\right) \operatorname{erf} i+\left(\mathscr{H}_{i} / \sqrt{ } \pi\right) \exp \left(\mathscr{M}_{i}{ }^{2}\right)\right\} /\left(\mathscr{M}_{i}{ }^{2}+\frac{1}{2}\right) \\
& \rightarrow\left(\mathscr{M}_{i}^{2}-\frac{1}{2}\right) /\left(\mathscr{M}_{i}{ }^{2}+\frac{1}{2}\right) \text { for large } \mathscr{M}_{i}
\end{aligned}
$$

This result is in substantial agreement with that obtained by Byutner. (16) Byutner's analysis demonstrated as we have, that for the heavy constitutents with molecular weight near the mean molecular weight, the collisional production is very nearly equal to the value for the undistributed Maxwellian condition.

In the case of the light-weight constitutents, the average relaxation rate parameter had to be evaluated numerically, where the integrations over $\alpha$ and $\varphi$ were carried out analytically and an integration on $v$ was performed using a simple quadrature. Figure 4 shows the 
relaxation rate parameters for the various binary gas mixtures one might expect in planetary atmospheres. For the terrestrial atmosphere, where hydrogen is minor constituent in an atomic oxygen background, relaxation occurs in between 3 and 8 collisions, depending on the temperature.

\section{(c) Evaluation of the distribution function}

The distribution function for particles with energy sufficient to escape (i.e. $E>0$ ) is determined by equations (28-31). Introducing the relaxed Lorentz-gas collision integrals given in equations (40) and (41) into equations (28-31), one obtains

$$
\begin{aligned}
\frac{f_{i}(r, E, \mu)}{F_{i}(E)}= & -\int_{r}^{\infty}\left\{\frac{1-\eta}{2} \int_{-1}^{+1} \frac{f_{i}\left(r^{\prime}, E, \mu\right)}{F_{i}(E)} d \mu+\eta\right\} \frac{d}{d r^{\prime}} \exp \left(-\int_{r}^{r^{\prime}} Q d \rho\right) d r^{\prime}-1<\mu<0 \\
= & \int_{1}^{r}\left\{\frac{1-\eta}{2} \int_{-1}^{+1} \frac{f_{i}\left(r^{\prime}, E, \mu\right)}{F_{i}(E)} d \mu+\eta\right\} \frac{d}{d r^{\prime}} \\
& \times \exp \left(-\int_{r^{\prime}}^{r} Q d \rho\right) d r^{\prime}+\exp \left(-\int_{1}^{r} Q d \rho\right) \quad \mu_{c}(1, r, E)<\mu<+1 \\
= & \int_{r_{p}}^{r}\left\{\frac{1-\eta}{2} \int_{-1}^{+1} \frac{f_{i}(r, E, \mu)}{F(E)} d \mu+\eta\right\} \frac{d}{d r^{\prime}} \exp \left(-\int_{r^{\prime}}^{r} Q d \rho\right) d r^{\prime} \\
& -\exp \left(-\int_{r_{p}(r, E, \mu)}^{r} Q d \rho\right) \int_{r_{p}\left(r, E, \mu^{\prime}\right)}^{\infty}\left\{\frac{1-\eta}{2} \int_{-1}^{+1} \frac{f_{i}(r, E, \mu)}{F_{i}(E)}+\eta\right) \\
& \times \frac{d}{d r^{\prime}}\left(-\int_{r_{p}(r, E, \mu)}^{r^{\prime}} Q d \rho\right) d r^{\prime} \quad 0<\mu<\mu_{c}(1, r, E)
\end{aligned}
$$

where

$$
\mu=\mathbf{r} \cdot \mathbf{V} /(|\mathbf{r}||\mathbf{V}|)
$$

and

$$
\mu_{0}(\rho, r, E)=\left\{1-(\rho / r)^{2}(2 E+1 / \rho) /(2 E+1 / r)\right\}^{1 / 2}
$$

and where

$$
r_{p}(r, E, \mu)=(-1 / 4 E)\left[1-\sqrt{ }\left(1+8 E r^{2}\left(1-\mu^{2}\right)(2 E+1 / r)\right)\right]
$$

and

$$
Q=\pi\left(d_{i B}{ }^{* 2} N_{B}^{*}(r) R^{*}\right) /\left[1-(r / \rho)^{2}\left(1-\mu^{2}\right)(2 E+1 / r) /(2 E+1 / \rho)\right]^{1 / 2}
$$

Here $\mu_{c}\left(\rho_{i} r, E\right)$ is that value for $\mu$ which will result in a trajectory with perigee radius $\rho$ for a particle originally at $r$ with energy $E$, and $r_{p}(r, E, \mu)$ is the perigee distance for a given trajectory. This set of rather formidable relations determines the distribution function once the integral

$$
\mathscr{L}(r, E)=(1 / 2) \int_{-1}^{+1}\left[f_{i}(r, E, \mu) / F_{i}(E)\right] d \mu
$$


is known. Thus, taking the integral of equation (54) over $\mu$ one obtains the single integral equation fo $\mathscr{L}$ given below

$$
\begin{gathered}
\mathscr{L}(r, E)=(-1 / 2) \int_{-1}^{0}\left\{\int_{r}^{\infty}\left[(1-\eta) \mathscr{L}\left(r^{\prime}, E\right)+\eta\right] \frac{d}{d r^{\prime}} \exp \left(-\int_{r}^{r^{\prime}} Q d \rho\right) d r^{\prime}\right\} d \mu \\
+(1 / 2) \int_{\mu_{c}(1, r, E)}^{+1}\left\{\int_{1}^{r}\left[(1-\eta) \mathscr{L}\left(r^{\prime}, E\right)+\eta\right) \frac{d}{d r^{\prime}} \exp \left(-\int_{r^{\prime}}^{r} Q d \rho\right) d r^{\prime}\right. \\
\left.+\exp \left(-\int_{1}^{r} Q d \rho\right)\right\} d \mu+(1 / 2) \int_{0}^{\mu_{c}(1, r, E)}\left\{\int_{r_{p}(r, e, \mu)}^{r}\left[(1-\eta) \mathscr{L}\left(r^{\prime}, E\right)+\eta\right]\right. \\
\frac{d}{d r^{\prime}} \exp \left(-\int_{r^{\prime}}^{r} Q d \rho\right) d r^{\prime} \\
\left.-\exp \left(-\int_{r_{p}(r, e, \mu)}^{r} Q d \rho\right) \int_{r_{p}(r, E, \mu)}^{\infty}\left[(1-\eta) \mathscr{L}\left(r^{\prime}, E\right)+\eta\right] \frac{d}{d r^{\prime}} \exp \left(-\int_{r_{p}(r, E, \mu)}^{r^{\prime}} Q d \rho\right) d r^{\prime}\right\} d \mu
\end{gathered}
$$

Or by inverting the order of the integrations

$$
\mathscr{L}(r, E)=\int_{+1}^{\infty}\left[(1-\eta) \mathscr{L}\left(r^{\prime}, E\right)+\eta\right] W\left(r^{\prime}, r\right) d r^{\prime}+A(r, E)
$$

where:

Case A. For $r^{\prime} \geq r$

$$
\begin{aligned}
W\left(r^{\prime}, r\right) & =(1 / 2)\left\{\int_{-1}^{0} \frac{d}{d r^{\prime}} \exp \left(-\int_{r}^{r^{\prime}} Q d \rho\right) d \mu+\int_{0}^{\mu_{c}(1, r, E)} \frac{d}{d r^{\prime}}\right. \\
& \times \exp \left(-\int_{r_{p}(r, E, \mu)}^{r^{\prime}} Q d \rho-\int_{r_{p}(r, E, \mu)}^{r} Q d \rho\right) d \mu
\end{aligned}
$$

Case B. For $r^{\prime} \leq r$

$$
\begin{aligned}
W\left(r^{\prime}, r\right)= & (1 / 2)\left\{\int_{\mu_{o}\left(r^{\prime}, r, E\right)}^{+1} \frac{d}{d r^{\prime}} \exp \left(-\int_{r^{\prime}}^{r^{\prime}} Q d \rho\right) d \mu-\int_{\mu_{o}\left(r^{\prime}, r, E\right)}^{\mu_{c}(1, r, E)} \frac{d}{d r^{\prime}}\right. \\
& \left.\times \exp \left(-\int_{r_{p}(r, E, \mu)}^{r^{\prime}} Q d \rho-\int_{r_{p}(r, E, \mu)}^{r} Q d \rho\right) d \mu\right\}
\end{aligned}
$$

and

$$
A(r, E)=(1 / 2) \int_{\mu_{0}(1, r, E)}^{+1} \exp \left(-\int_{1}^{r} Q d \rho\right) d \mu
$$

Thus, the equation for $\mathscr{L}$ is a linear integral equation of the second kind and may be solved in the most general case using one of the many numerical techniques available. ${ }^{(38)}$ However, before carrying out a numerical analysis of the equation it is well to consider the simplifications which may be applied when dealing with a planetary atmosphere. First, the transition region of the atmosphere of a planet such as Earth or Mars is confined to a very thin layer in comparison with the radius of the planet. Second, the radius, $r$, is not a natural coordinate to use to study the collisional effects. We decided that a more convenient parameter relating to the collisional transition would be the parameter ${ }^{(16)}$

$$
y=\int_{r}^{\infty}\left[\nu_{i}(\rho) / V(\rho)\right] d \rho
$$


which is the natural logarithm of the probability that a particle with velocity $V$ directed along the radius vector will escape from the atmosphere. Notice the similarity here to the "optical depth" used in the radiative transfer problem

Furthermore, since the transition from the collision-dominated region to the collisionless exosphere must occur in a layer, thin compared to the radius of the planet, it is assumed that the background gas density may be approximated by the simple exponential

$$
N_{B}^{*}(r)=N_{B}^{*}(R) \exp \left[-\mathscr{H}_{B}^{2}(r-1)\right]
$$

Thus, for hard spherical scatterers

and the inverse

$$
\begin{aligned}
y & =\left[\pi\left(d_{i B}{ }^{* 2} N_{B}{ }^{*}(R) R^{*}\right) / \mathscr{M}_{B}{ }^{2}\right] \exp \left[-\mathscr{M}_{B}{ }^{2}(r-1)\right] \\
& =y_{0} \exp \left[-\mathscr{M}_{B}{ }^{2}(r-1)\right]
\end{aligned}
$$

$$
r=1-\log \left(y / y_{0}\right) / \mathscr{M}_{B}^{2}
$$

where $y_{0}$ is the value of $y$ at $r=1$. Now neglecting the square of $(r-1)$ compared with unity, the integral where

$$
I\left(y_{1}, y_{2}, \mu\right)=\int_{r_{1}}^{r_{2}} Q d \rho=(\sqrt{\pi} Z / \mu) y_{1} \exp \left(Z^{2}\right)\left\{\operatorname{erfc}(z)-\operatorname{erfc}\left[Z^{2}-\log \left(y_{2} / y_{1}\right)\right]^{1 / 2}\right\}
$$

and where

$$
Z=\left[\lambda \mu^{2} /\left(1-\mu^{2}\right)\right]^{1 / 2}
$$

$$
\lambda=\mathscr{K}_{B}^{2}(2 E+1) /(4 E+1)
$$

Notice that $\lambda$, henceforth designated as the curvature parameter, can be expressed in terms of the scale height of the background gas, the particle's energy, and the radius of the planet

$$
\lambda=\left(R^{*} / H_{B}{ }^{*}\right)(2 E+1) /(4 E+1)
$$

where $R^{*}$ and $H_{B}{ }^{*}$ are dimensional quantities. The scale height $H_{B}{ }^{*}$ has the definition

$$
H_{B}{ }^{*}=\left(k T^{*} / m_{B}{ }^{*} g^{*}\right)
$$

where $g^{*}$ is the gravitational acceleration at the distance $R^{*}$, and $T^{*}$ the temperature of the background gas. For the terrestrial atmosphere $\lambda$ varies between 25 and 150 depending on the temperature and on the energy of the particles under discussion.

The integral equation for $\mathscr{L}$ now takes the form

where:

$$
\mathscr{L}(y, \lambda)=\int_{0}^{y_{0}}\left[(1-\eta) \mathscr{L}\left(y^{\prime}, \lambda\right)+\eta\right] \omega\left(y^{\prime}, y\right) d y^{\prime}+\mathcal{A}(y, \lambda)
$$

Case $A$. For $y^{\prime} \leq y$

$$
\begin{aligned}
\omega\left(y^{\prime}, y\right)=(-1 / 2)\left\{\int_{\mu_{o}\left(y^{\prime}, y, \lambda\right)}^{1} \frac{d}{d y^{\prime}} \exp \left[-I\left(y, y^{\prime}, \mu\right)\right] d \mu-\int_{\mu_{\mathrm{c}}\left(y, y^{\prime}, \lambda\right)}^{\mu_{\mathrm{e}}\left(y_{0}, y, \lambda\right)} \frac{d}{d y^{\prime}}\right. \\
\left.\times \exp \left[-I\left(y \cdot y^{\prime}, \mu\right)-2 I\left(y_{y}, y, \mu\right)\right] d \mu\right\}
\end{aligned}
$$

Case B. For $y^{\prime} \leq y$

$$
\begin{aligned}
\omega\left(y^{\prime}, y\right)=(1 / 2)\left\{\int_{-1}^{0} \frac{d}{d y^{\prime}} \exp \left[-I\left(y, y^{\prime}, \mu\right)\right] d \mu+\int_{0}^{\mu_{0}\left(y_{0}, y, \lambda\right)} \frac{d}{d y^{\prime}}\right. \\
\left.\times \exp \left[-I\left(y, y^{\prime}, \mu\right)-2 I\left(y_{p}, y, \mu\right)\right]\right\} d \mu
\end{aligned}
$$


and where

and

$$
\begin{gathered}
\mathcal{A}(y, \lambda)=(1 / 2) \int_{\mu_{0}\left(y_{0}, y, \lambda\right)}^{+1} \exp \left[-I\left(y_{0}, y, \mu\right)\right] d \mu \\
\mu_{0}\left(y^{\prime}, y, \lambda\right)=\left\{\log \left(y^{\prime} / y\right) /\left(\lambda+\log \left(y^{\prime} / y\right)\right)\right\}^{1 / 2}
\end{gathered}
$$

$$
y_{p}=y \exp \left[\lambda \mu^{2} /\left(1-\mu^{2}\right)\right]
$$

The quantity $y_{p}$ is the value of $y$ at $r=r_{p}$, the perigee point.

It is interesting to notice that when $\lambda \rightarrow \infty$, that is, when the planet is treated as being of infinite radius, the weighting function takes on a simple form and $\mathscr{L}$ satisfies the equation

$$
\begin{gathered}
\mathscr{L}(y, \infty)=(1 / 2)\left\{\int_{0}^{y_{0}}\left[(1-\eta) \mathscr{L}\left(y^{\prime}, \infty\right)+\eta\right]\left[-E_{i}\left(-\left|y-y^{\prime}\right|\right)\right] d y+\exp \left[-\left(y_{0}-y\right)\right]\right. \\
\left.+\left(y_{0}-y\right) E_{i}\left(-\left|y_{0}-y\right|\right)\right\}
\end{gathered}
$$

where

$$
-E_{i}(-Z)=\int_{Z}^{\infty}[\exp (-t) / t] d t
$$

is the exponential integral. It is interesting to note the similarity of equation (79) to the basic equations for scattering of light in a plane parellel atmosphere. When $\lambda \rightarrow \infty$, equation (79) for $\mathscr{L}$ is correct for any spatial distribution of background gas so long as the integral defining $y$ is single valued.

Once $\mathscr{L}$ is determined from these equations, the solution for the distribution function is easily evaluated from the following equations:

$$
\begin{aligned}
& \frac{f_{i}(y, \lambda, \mu)}{F_{\imath}(E)}= \int_{0}^{y}\left[(1-\eta) \mathscr{L}\left(y^{\prime}, \lambda\right)+\eta\right] \frac{d}{d y^{\prime}} \exp \left[-I\left(y, y^{\prime}, \mu\right)\right] d y^{\prime} \quad-1<\mu<0 \\
&=-\int_{y}^{y_{0}}\left[(1-\eta) \mathscr{L}\left(y^{\prime}, \lambda\right)+\eta\right] \frac{d}{d y^{\prime}} \exp \left[-I\left(y^{\prime}, y, \mu\right)\right] d y^{\prime} \\
&+\exp \left[-I\left(y_{0}, y, \mu\right)\right] \\
&=-\int_{y}^{y_{0}}\left[(1-\eta) \mathscr{L}\left(y^{\prime}, \lambda\right)+\eta\right] \frac{d}{d y^{\prime}} \exp \left[-I\left(y^{\prime}, y, \mu\right)\right] d y^{\prime} \\
&-\int_{0}^{y_{y}}\left[(1-\eta) \mathscr{L}\left(y^{\prime}, \lambda\right)+\eta\right] \frac{d}{d y^{\prime}} \exp \left[-I\left(y_{p}, y, \mu\right)-I\left(y_{p}, y^{\prime}, \mu\right)\right] \\
& 0<\mu^{\prime}, \mu<\mu\left(y_{0}, y, \lambda\right)
\end{aligned}
$$

(d) Molecular escape flux

The primary concern of this study is the escape flux. This flux is obtained from the distribution by multiplying the radial velocity of the particles and summing over all particles as follows:

$$
\mathscr{F}=4 \pi r^{2} \overline{n u_{r}}=8 \pi^{2} r^{2} \int_{-1 / 2^{+}}^{\infty}(2 E+1 / r) F_{i}(E) \int_{-1}^{+1} \frac{f_{i}(y, E, \mu)}{F_{i}(E)} \mu d \mu d E
$$


In this expression the quantity $f_{i}(y, E, \mu) / F_{i}(E)$ is determined from equation (82) for $E>0$, but nothing has been said about the lower energy particles. The distribution function in this range must be known in order to complete the analysis.

In the lower regions of the atmosphere, where collisions occur very frequently, the distribution function must be nearly Maxwellian and consistent with the results derived from the Chapman-Enskog theory. ${ }^{(29)}$ Thus, the upward flux of material caused by the escape of molecules is represented in the lower region by a distortion of the Maxwellian distribution throughout the entire velocity range from zero to the largest velocities. This process is the ordinary macroscopic diffusion. However, as the altitude increases such that particles can travel ballistically without suffering collision, a radical change must occur in this process. In this region of the atmosphere the distribution function for particles with energy less than zero (i.e. velocity less than the escape velocity) can exhibit no net outward motion. This results from the fact that in collisionless flow the distribution function is constant along a trajectory, and all trajectories for particles with $E<0$ cross each height twice, once on leaving and once on re-entering the lower atmosphere. Consequently, the escape flux is entirely determined from the distribution function for $E>0$ as $y \rightarrow 0$. That is

$$
\mathscr{F}=8 \pi^{2} \lim _{y \rightarrow \infty} r^{2}(y) \int_{0}^{\infty}(2 E+1 / r) F_{i}(E) \int_{-1}^{+1}\left[f_{i}(y, E, \mu) / F_{i}(E)\right] \mu d \mu d E
$$

The ratio of the escape flux determined from equation (83) to the result obtained from the collisionless theory is

where

$$
\mathscr{F} \mid \mathscr{F}_{\text {C.L. }}=\left[2 \mathscr{M}_{i}{ }^{4} /\left(\mathscr{K}_{i}{ }^{2}+1\right)\right] \int_{0}^{\infty}(2 E+1) \exp \left(-2 \mathscr{K}_{i}{ }^{2} E\right)\left(\delta \mathscr{F} / \delta \mathscr{F}_{\text {C.L. }}\right) d E
$$

$$
\frac{\delta \mathscr{F}}{\delta \mathscr{F}}=\lim _{\text {c.I. }}\left[\frac{r^{2}(y)(2 E+1 / r(y))}{2 E+1}\left\{2 \int_{-1}^{+1}\left(f_{i} / F_{i}\right) \mu d \mu\right\}\right]
$$

is the ratio of the escape flux at a specific energy to the corresponding escape flux in the collisionless approximation. The flux ratio can be written in terms of $\mathscr{L}$ as follows:

$$
\begin{aligned}
\frac{\delta \mathscr{F}}{\delta \mathscr{F}_{\text {C.I. }}=\lim _{y \rightarrow 0}\left[\frac { r ^ { 2 } ( y ) ( 2 E + 1 / r ( y ) ) } { 2 E + 1 } \left\{\int_{0}^{y_{0}}\left[(1-\eta) \mathscr{L}\left(y^{\prime}, \lambda\right)+\eta\right] \omega_{\mathcal{F}}\left(y^{\prime}, y\right) d y^{\prime}\right.\right.} \\
\left.\left.+2 \int_{\mu_{0}\left(y_{0}, y, \lambda\right)}^{1} \exp \left[-I\left(y_{0}, y, \mu\right)\right] \mu d \mu\right\}\right]
\end{aligned}
$$

where the weighting function $\omega_{\mathcal{F}}$ has the value:

Case $A$. For $y^{\prime} \geq y$

$$
\begin{aligned}
\omega_{F^{F}}\left(y^{\prime}, y\right)=-2 \int_{\mu_{0}\left(y^{\prime}, y, \lambda\right)}^{1} & \mu \frac{d}{d y^{\prime}} \exp \left[-I\left(y^{\prime}, y, \mu\right)\right] d \mu \\
& +2 \int_{\mu_{0}\left(y^{\prime}, y, \lambda\right)}^{\mu_{0}\left(y_{0}, y, \lambda\right)} \mu \frac{d}{d y^{\prime}} \exp \left[-I\left(y, y^{\prime}, \mu\right)+2 I\left(y_{p}, y, \mu\right)\right] d \mu
\end{aligned}
$$

Case B. For $y^{\prime} \leq y$

$$
\begin{aligned}
\omega_{\mathscr{F}}\left(y^{\prime}, y\right)=2 \int_{-1}^{0} \mu & \frac{d}{d y^{\prime}} \exp \left[-I\left(y, y^{\prime}, \mu\right)\right] d \mu \\
& +2 \int_{0}^{\mu_{0}\left(y_{0}, y, \lambda\right)} \mu \frac{d}{d y^{\prime}} \exp \left[-I\left(y, y^{\prime}, \mu\right)+2 I\left(y_{p}, y, \mu\right)\right] d \mu
\end{aligned}
$$


For the planar approximation these equations may be integrated to yield the results:

Case $A$. For $y^{\prime} \geq y$

$$
\omega_{\mathfrak{F}}\left(y^{\prime}, y\right)=2\left\{\exp \left(-y^{\prime}+y\right)+\left(y^{\prime}-y\right) E_{\imath}\left(-y^{\prime}+y\right)\right\}
$$

Case B. For $y^{\prime} \leq y$

and

$$
\omega_{\mathscr{F}_{F}}\left(y^{\prime}, y\right)=2\left\{\exp \left(-y+y^{\prime}\right)+\left(y-y^{\prime}\right) E_{i}\left(-y+y^{\prime}\right)\right\}
$$

$$
\begin{aligned}
& 2 \int_{\mu_{\mathrm{c}}\left(y_{0}, y, \infty\right)}^{1} \exp \left[-I\left(y_{0}, y, \mu\right)\right] \mu d \mu=\left(1-y_{0}+y\right) \\
& \times \exp \left(-y_{0}+y\right)-\left(y_{0}-y\right)^{2} E_{2}\left(-y_{0}+y\right) .
\end{aligned}
$$

\section{RESULTS AND DISCUSSION}

The theoretical results derived above can answer a number of fundamental questions concerning the rate of escape of atmospheric gases and the nature of the outermost atmosphere. The discussion presented here will be limited to the major constituent gas and to minor constituent gases of relatively low molecular weight. These two cases illustrate the major physical phenomena associated with the escape of atmospheric gases; in addition, they are the most interesting from the geophysical point of view.

\section{(a) Major constituents}

When the molecular weight of the minor constituent gas is equal to the mean molecular weight of the atmosphere the average relaxation rate parameter is close to unity (see Fig. 4). Byutner ${ }^{(16)}$ has pointed out that for the escape problem the major constituent also exhibits nearly immediate relaxation to the Maxwellian state. One would of course expect this behavior from previous studies of translational relaxation in gases ${ }^{(30)}$ In the atmospheric model postulated here this immediate relaxation to Maxwellian is represented by setting the average relaxation rate parameter equal to unity. As a consequence of this simplification one can represent the distribution function for the major constituent by a relatively simple expression: introducing the condition $\eta=1$ into equation (81) and simplifying, one obtains

$$
\frac{f_{i}(y, E, \mu)}{F_{2}(E)}= \begin{cases}1 & \mu_{c}\left(y_{0}, y, \mu\right)<\mu<+1 \\ 1-\exp \left[-I\left(y_{p}, y, \mu\right)-I\left(y_{p}, 0, \mu\right)\right] & 0<\mu<\mu_{c}\left(y_{0}, y, \lambda\right) \\ 1-\exp [-I(y, 0, \mu)] & -1<\mu<0\end{cases}
$$

where $I$ is defined by equation (68), $y_{p}$ is the value of $y$ at perigee of the characteristic and $\lambda$ is the curvature parameter. Notice that this expression applies to particles with velocity in excess of the escape speed only.

The ability to obtain a simple analytic solution for this example is of particular interest since many features of the transition zone can be uncovered which would be difficult to obtain numerically. Let us begin this examination of the transition region by considering the manner in which the distribution function changes with height and zenith angle. One expects the distribution function to be Maxwellian at low altitudes, i.e. large $y$, and to decrease for particles with velocity directed toward the earth as higher altitudes are considered. This expectation is indeed correct, as the results shown in Fig. 5 illustrate. These data for various values of the height parameter $y$ show the variation of the distribution 
function with the cosine of the zenith angle (i.e. $\mu$ ). Notice that the distribution is initially Maxwellian, then exhibits a transition to a nearly discontinuous function similar to the collisionless solution determined from the critical level theory. It is of particular interest to determine the critical level which would simulate the exact solution at great heights. This can be done by considering the behavior of $f_{i}$ as $y \rightarrow 0$, where

$$
\frac{f_{i}(y, E, \mu)}{F_{i}(E)}=1-\exp \left[-2 I\left(y_{p}, 0, \mu\right)\right]
$$

The rapid change occurs where

$$
2 I\left(y_{p}, 0, \mu\right) \cong 2 \sqrt{ }(\pi \lambda) y_{p}=1
$$

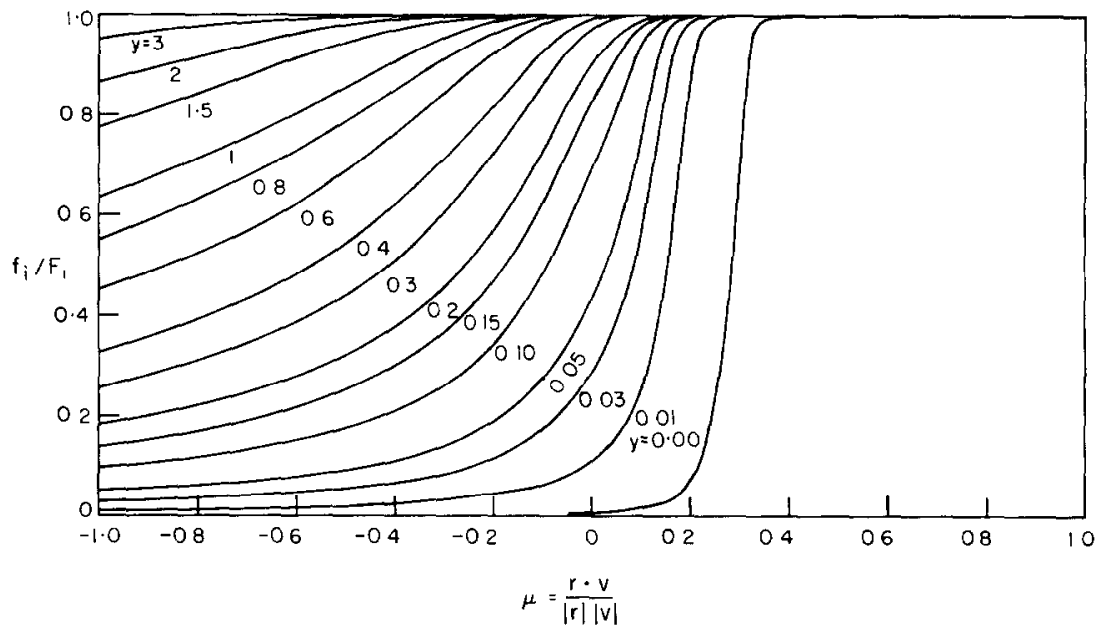

Fig. 5. Distribution fUNCtion fOR A MAJOR CONSTITUENT Gas WHEN THE CURVATURE PARAMETER $\lambda=50$.

a result which does not agree with the older heuristic theories. Recall that in an atmosphere where the collision frequency decreases exponentially with height, the parameter $y$ is the ratio of the scale height and local mean free path. Thus the present theory indicates that the critical height can be chosen at the level where the mean free path length is

$$
l^{*}=2 \sqrt{ }\left(\pi R^{*} H_{B}(2 E+1) /(4 E+1)\right)
$$

whereas the old heuristic theories chose the critical level to occur where

$$
l^{*}=H_{B}^{*}
$$

Consequently, the older theories predicted a critical level which occurred at a much too low altitude. Note that the critical level predicted by the present calculation is

$$
r_{\text {er }}=1+\log \left(2 \sqrt{ }(\pi \lambda) y_{0}\right) / \mathscr{M}_{B}{ }^{2}
$$

giving an error in height of the critical level for the old theories of

$$
\Delta h=\log (2 \sqrt{ }(\pi \lambda)) / \mathscr{H}_{B}^{2}
$$

this being of the order of $250 \mathrm{~km}$ for the Earth. 
The primary macroscopic quantity of interest here is the escape flux for the major constituent gas. One can easily determine this flux by introducing the distribution function given by equation (92) into equation (85) and integrating. After rearrangement one obtains

$$
\begin{aligned}
\frac{\delta \mathscr{F}}{\delta \mathscr{F}_{\mathrm{C} . \mathrm{L}}} & =1+\int_{0}^{\infty} \frac{4 E h+4 E+1}{2 E+1}\left\{1-\exp \left[-\xi_{0} \exp \left(-\mathscr{M}_{B}{ }^{2} h\right)\right]\right\} d h \\
& =1+\left[\gamma+\log \xi_{0}-E_{\imath}\left(-\xi_{0}\right)+\frac{E 4}{(4 E+1)} B^{2} \sum_{n=1}^{\infty} \frac{(-1)^{n+1}}{n^{2} n !} \xi_{0}^{n}\right] / \lambda
\end{aligned}
$$

where $h=r-1, \gamma$ is Euler's constant and $\xi_{0}=2 V^{\prime}(\pi \lambda) y_{0}$. This result can be integrated over all energies using equation (84) to give the total escape flux as a function of $y_{0}$ and exospheric temperature. This integration has been carried out using a simple quadrature

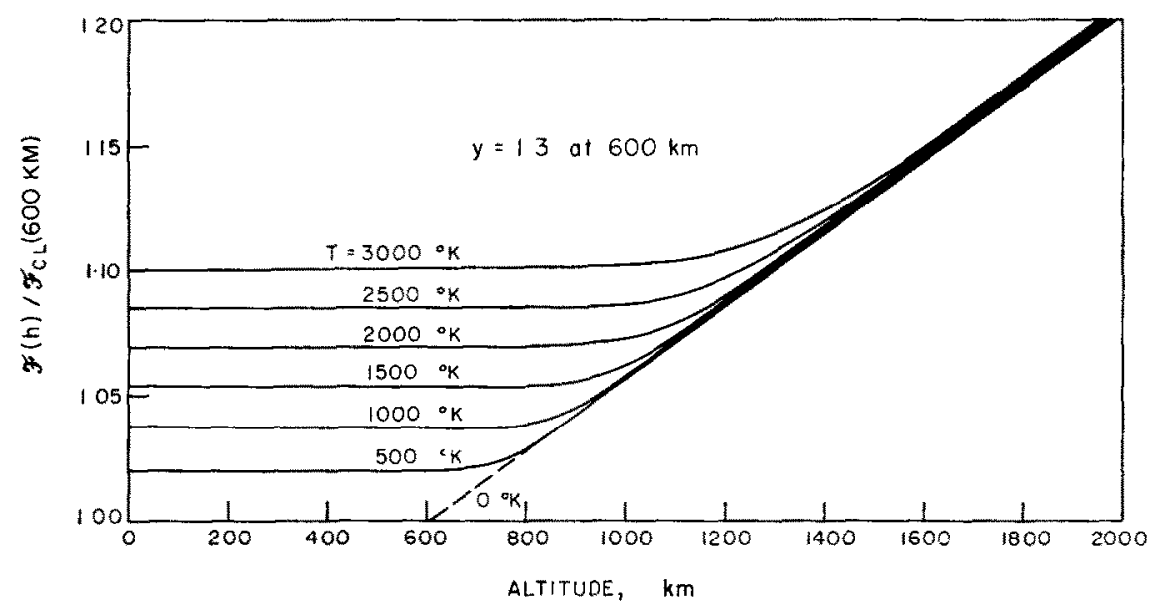

FIG. 6. THE VARIATION OF THE ESCAPE FLUX FOR A MAJUR CONSTTTUENT (ATOMIC OXYGEN IN THE EARTH'S ATMOSPHERE) WITH BOUNDARY LEVEL FOR VARIOUS EXOSPHERIC TEMPERATURES.

formula for various values of temperature in an atmosphere made up of atomic oxygen. The results of these computations are illustrated in Fig. 6. The escape flux is constant so long as the base level is deep in the lower atmosphere, but the flux changes to the collisionless value when the base level is chosen too high. This is of course the expected behavior.

The escape flux derived from the present theory is compared in Fig. 7 with the flux obtained from the classical critical level theory using $y_{\mathrm{cr}}=\left(2 \sqrt{ } \pi_{\mathscr{N}_{B}}\right)^{-1}$. This is the critical level for particle with zero energy. Observe that the results for escape flux agree very well using the new definition for the critical level. We thus conclude that the escape flux can be determined accurately for major constituents using the critical level concept if the critical level is defined as the level at which the mean horizontal free path has the value

$$
l^{*}=2 \sqrt{ }\left(\pi R^{*} H_{B}^{*}\right)
$$

One further point of interest in this development is that the results obtained by neglecting the curvature of the planet agree very well with the results obtained from the rigorous analysis. For this situation where the relaxation rate parameter is unity, the planar atmospheric model indicates that the escape flux is equal to the value given by the critical level theory. This is true so long as the criterion given by equation (99) is used to determine the 


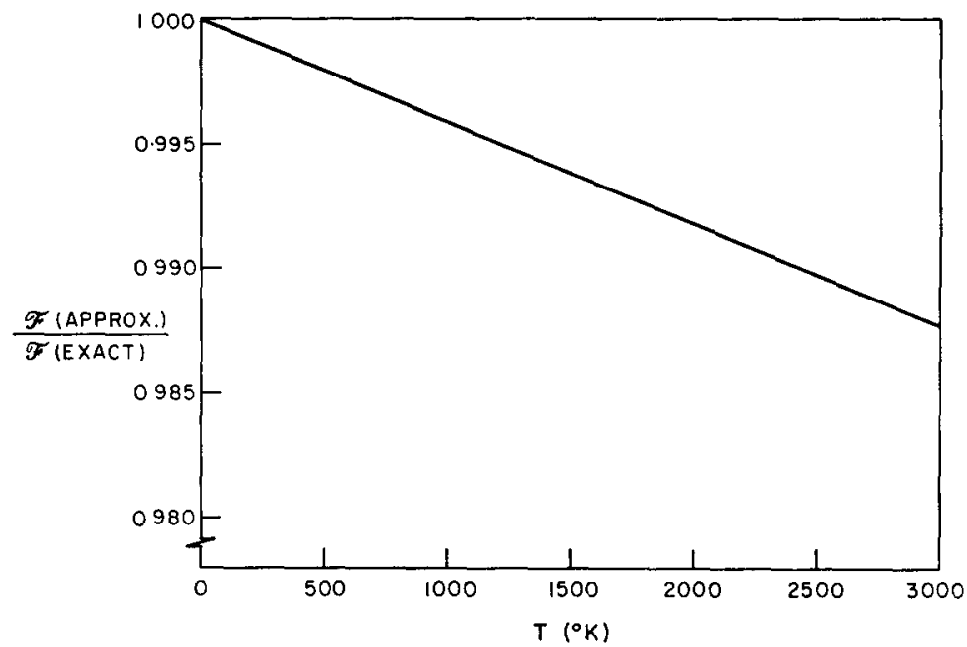

FIG. 7. A COMPARISON OF THE ESCAPE FLUX COMPUTED USING THE NEW CRITICAL LEVEL THEORY WITH THE EXACT COMPUTATION FOR ATOMIC OXYGEN IN THE EARTH'S ATMOSPHERE.

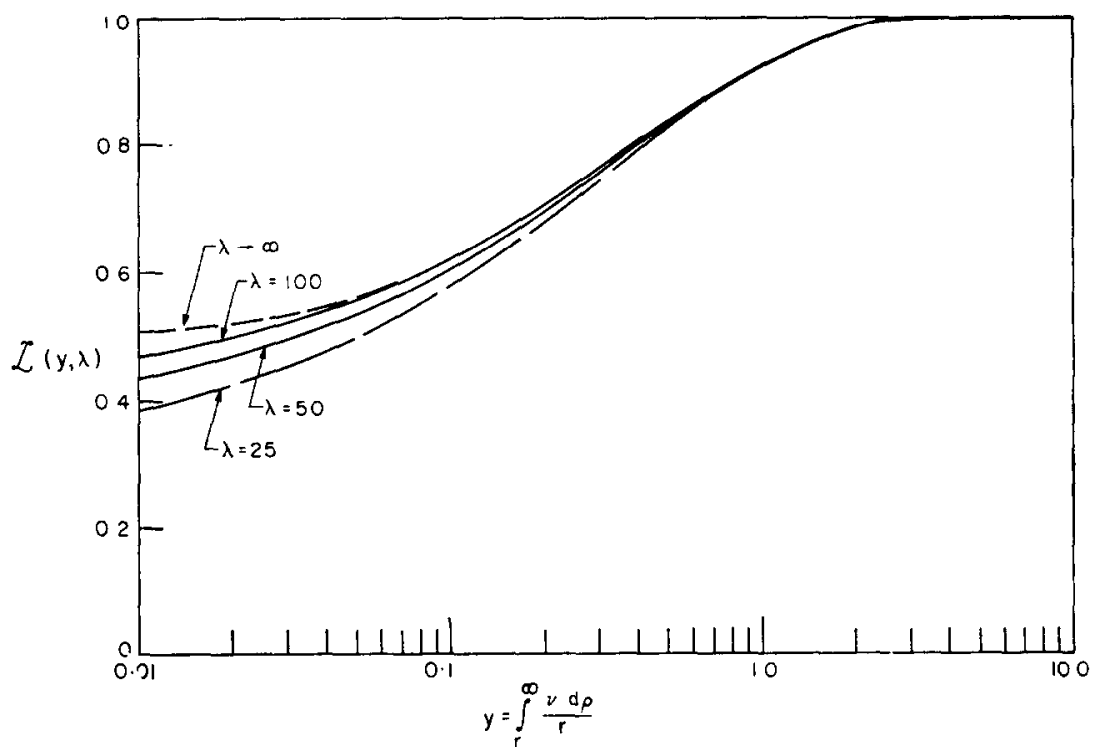

FIG. 8. A COMPARISON OF THE $L$ FUNCTION FOR THE PLANAR ATMOSPHERE WITH VARIOUS SPHERICAL ATMOSPHERES.

critical level. Second, when the mean value of the distribution function

$$
\mathscr{L}(y, \lambda)=(1 / 2) \int_{-1}^{+1}\left[f_{i}(y, \lambda, \mu) / F_{i}(E)\right] d \mu
$$

discussed in the previous section, is compared for the planar atmosphere and the curved atmosphere, for values of $\lambda$ of interest on the terrestrial planets the planar theory adequately represents the distribution function in the transition region. This comparison is illustrated in Fig. 8. These results strongly tempt one to conclude that the escape flux can be obtained in the most general case by neglecting the effects of curvature, while determining the effective area of the planetary atmosphere from a critical level concept similar to 
that derived above. This approach is valid when $\eta=1$, and it is physically reasonable to use the same technique for the more complex situation where the relaxation is not instantaneous.

\section{(b) Minor constituents with low molecular weight}

Light-weight gases such as hydrogen and helium have high thermal speeds and consequently they tend to escape rapidly from a planetary atmosphere. Bates and Patterson ${ }^{(34,35)}$ have pointed out that in order to sustain the great upward fluxes demanded by thermal escape, one must have large gradients in the number density at low altitudes. In practice these high gradients decrease with altitude and generally approach the normal exponential behavior of a quiescent atmosphere at altitudes near the transition level where the actual escape occurs. The result of this dependence of the minor constituents upon the escape flux is far-reaching.

The initial decrease in the density near the level of diffusive separation is strongly dependent upon the escape flux, the decrement increasing with increasing vertical flux, and consequently, the amount of hydrogen in the thermosphere as well as in the exosphere is determined by the rate at which material is lost from the atmosphere. The formalism necessary for an analysis of the escape of a minor constituent gas has been developed and can now be used to determine these rates accurately.

The distribution function for a minor constituent gas obeys the integral equations derived above and summarized in equations (73) through (81). These equations are linear integral equations of the second kind and are readily solvable using standard techniques. In general they can be solved by assuming a set of discrete points at which the function is to be determined, introducing a suitable interpolation, and then carrying out the integrations to yield a set of difference equations of the form

$$
\mathscr{L}_{j}=\sum_{i=0}^{N}\left[(1-\eta) \mathscr{L}_{i}+\eta\right] \omega_{i j}+A_{j} j-0,1, \ldots, N
$$

This system of linear algebraic equations can be inverted to yield a discrete set of values for $y_{i}$ and $\mathscr{L}_{i}$, which are sufficient to determine the distribution function and the escape flux that we require. The numerical inversions were carried out using the IBM $7090 \mathrm{com}$ puter to simulate the terrestrial atmosphere for the case where the curvature parameter tends to infinity.

Figure 9 illustrates the results of the numerical evaluation of $\mathscr{L}$ for various values of the relaxation rate parameter. Notice that $\mathscr{L}$ is a monotonically decreasing function of both $y$ and $\eta$. This is of considerable interest since $\mathscr{L}$ is analogous to the number density, and a decrease below the Maxwellian value of 1 indicates an effect similar to the reduction of the number density due to diffusion which Bates has discussed for the thermosphere. The reduction of $\mathscr{L}$ with decreasing $\eta$ illustrates the increase in the resistance to any net motion which is a consequence of increasing the relative mass of the background scatters. The reduction of the number of particles present at escape velocities as height is increased is seen clearly in Fig. 10, which shows the variation of the distribution function with $y$ when $\eta=0.25$, a typical value for hydrogen in the Earth's atmosphere. It is equally evident that the vertical flux of escaping material should be reduced as $\eta$ decreases. This effect is illustrated in Fig. 11, which shows the variation of the escape flux with the relaxation rate parameter. Furthermore, the escape flux ratio tends to 1 as $\eta$ tends to unity as one would expect for the planar approximation, since every collisional loss is exactly balanced by a corresponding gain when $\eta=1$. 
The variation of the escape flux for hydrogen and helium in the Earth's atmosphere is illustrated in Fig. 12 for the range of temperatures one would expect for the terrestrial exosphere. The variation in the flux shown in Fig. 12 indicates that the classical collisionless theory gives nearly the correct variation with temperature, but that the overall level of the

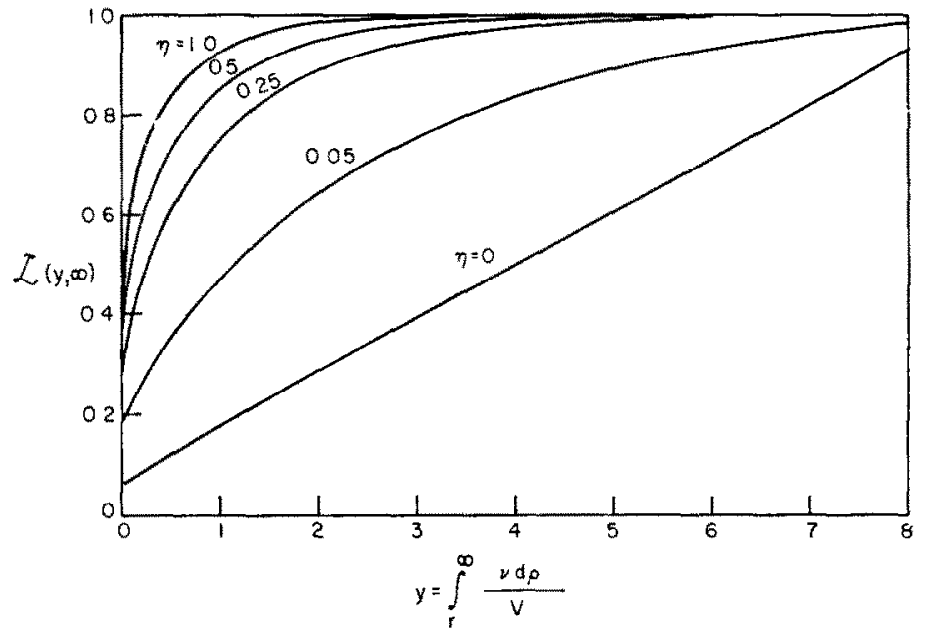

Fig. 9. THE VARIATIONS OF the $L$ FUNCTION WITH THE RELAXATION RATE PARAMETER AND $y$ FOR THE PLANAR ATMOSPHERE $(\lambda \rightarrow \infty)$.

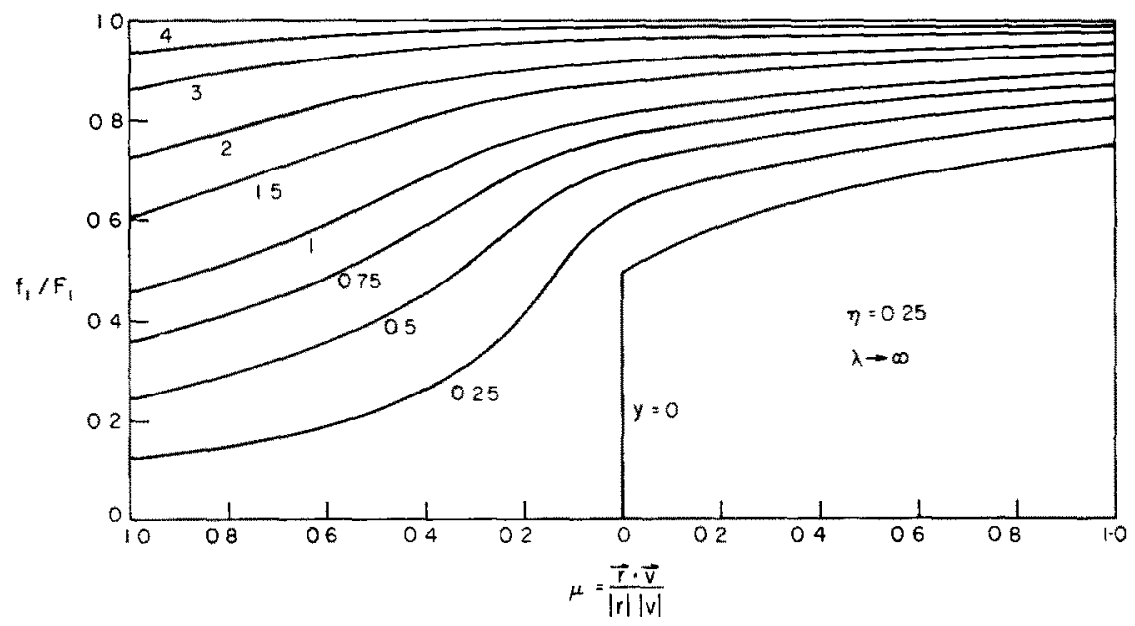

FIG. 10. DISTRIBUTION FUNCTION FOR A MINOR CONSTITUENT (HYDROGEN IN THE EARTH's ATMOSPHERE) WITH $\eta=0.25$ FOR THE PLANAR ATMOSPHERE $(\lambda \rightarrow \infty)$.

flux is over-estimated when the resistance to vertical motion is neglected. The reduction in the escape flux indicated by the present theory is by no means trivial, since a decrease in the flux of the order indicated in Fig. 12 will greatly increase the amount of hydrogen present at low levels, and will decrease the hydrogen in the outermost exosphere in direct proportion to the fiux reduction. 


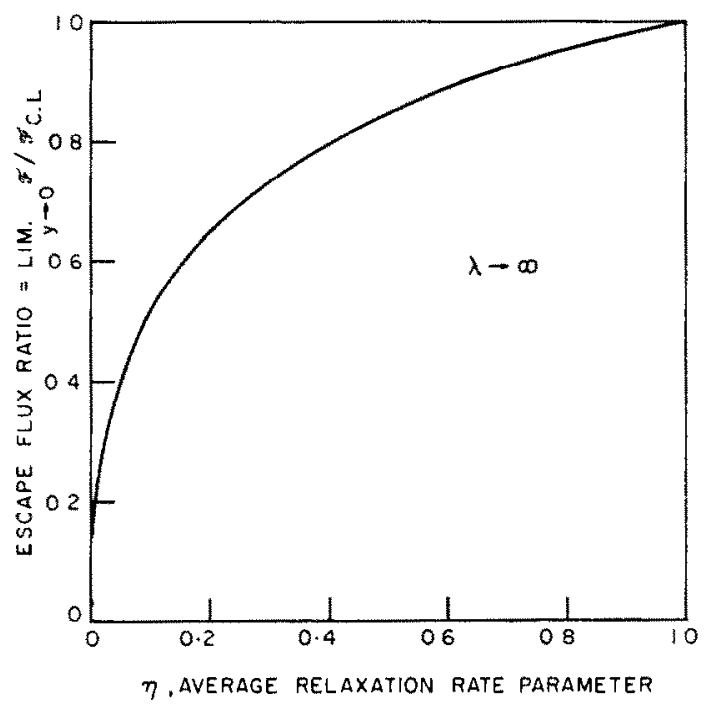

Fig. 11. ESCAPE FLUX RATIO AS A FUNCTION OF THE RELATION RATE PARAMETER FOR THE PLANAR ATMOSPHERE $(\lambda \rightarrow \infty)$.

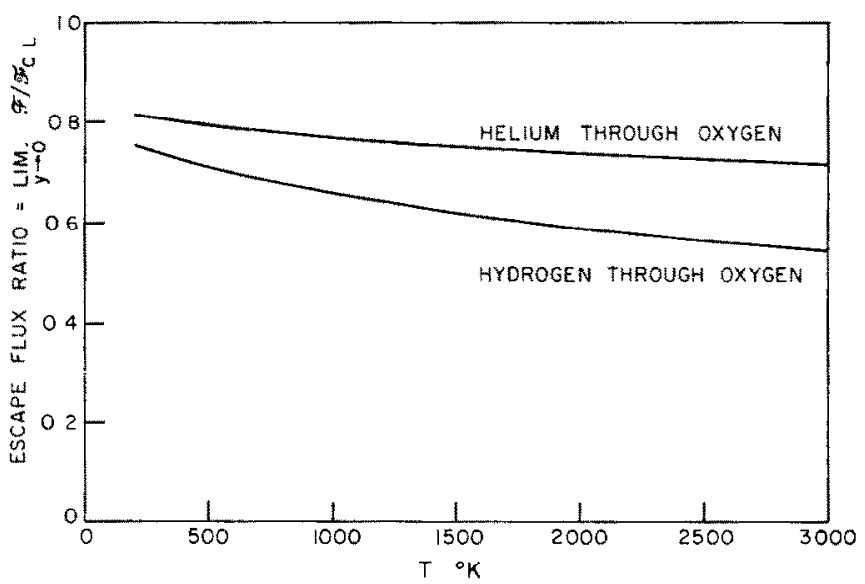

FIG. 12. ESCAPE FLUX RATIO AS A FUNCTION OF EXOSPHERIC TEMPERATURE FOR VARIOUS GAS MIXTURES EXPECTED IN THE EARTH'S ATMOSPHERE.

\section{CONCLUSION}

The classical problem of determining the rate of escape of a planetary atmosphere has been re-examined from the point of view of the kinetic theory of gases. This analysis, based upon a relaxed Lorentz-gas collision model, indicates that in the transition zone between the collisionless exosphere and the collision-dominated lower atmosphere, the molecular collisions act in two ways to effect the outermost atmosphere. First, an effective evaporative surface, in concept similar to the apparent photospheric surface of the sun, is determined by collisions of molecules moving tangential to the atmosphere. This effective surface or critical layer occurs where the local mean free path has the value

$$
l^{*}=2 \sqrt{ }\left(\pi R^{*} H_{B}{ }^{*}\right),
$$


a result in disagreement with the previous heuristic theories. Second, the total amount of material passing through the transition region is determined by a diffusion-like process which is dependent upon the frequency of collisions, and the rate at which distortions in the distribution function from Maxwellian are destroyed by collisions. The net effect of collisions is to decrease the upward or escape flux of material and thus reduce the density of the outermost atmosphere of a planet. The reduction of the escape flux is highly dependent upon the molecular weights of the escaping and the background gases. When the background gas is very heavy compared to the escaping gas the escape flux is greatly reduced, but when the molecular weight of the escaping gas is equal to that for the background, the escape flux is equal to that predicted by the critical level theory using the height criterion derived in this paper.

These two characteristics of the thermal escape of gases are to be expected: the first from the rapid vertical decay of the collision frequency which has been used in the earlier theories to justify the critical level concept; the latter diffusion effect is of course physically analogous to the diffusing atmosphere introduced by Bates. ${ }^{(33)}$ However, in this transition region one cannot treat the gases from the continuum point of view.

Acknowledgements--This work was supported in part by the Upper Atmospheric Physics Laboratory, Geophysics Research Directorate, AFCRL, by the Phoenix Memorial Research Grant of the University of Michigan, and by NASA Contract No. NASA - 54(05).

\section{REFERENCES}

1. J. J. Waterston, Proc. Roy. Soc., Lond. 5, 604 (1846).

2. J. J. Waterston, Phil. Trans. Roy. Soc., Lond. A183, 1 (1892).

3. G. J. STONEY, Proc. Roy. Soc. Lond. 17, Sec. 4, 1 (1868).

4. G. J. STONRY, Astrophys. J. 7, 25 (1898).

5. G. J. STONEY, Astrophys. J. 11, 251 (1900).

6. G. J. STONEY, Astrophys. J. 11, 357 (1900).

7. G. J. STONEY, Astrophys. J. 12, 201 (1900).

8. G. J. STONEY, Astrophys. J. 20, 69 (1904).

9. S. R. Cook, Astrophys. J. 11, 36 (1900).

10. G. H. BRYAN, Phil. Trans. Roy. Soc., Lond. A196, 1 (1901).

11. R. EMDEN, Gas Kugeln, Teubner, Leipzig (1907).

12. E. A. MILNE, Trans. Camb. Phil. Soc. 22, (26) 483 (1923).

13. J. E. Jones, Trans. Camb. Phil. Soc. 22, (28) 535 (1923).

14. J. H. Jeans, The Dynamical Theory of Gases (4th ed.). Cambridge University Press, Cambridge (1925).

15. L. SPITZER, The Atmospheres of the Earth and Planets (ed. G. P. Kuiper, 2nd ed.), p. 211. University of Chicago Press, Chicago (1952).

16. E. K. BYUTNER, Astr. Zh. 35, 572 (1958).

17. E. K. BYu'Ner, Astr. Zh. 36, 89 (1959).

18. E. J. OPIK and S. F. SINGER, Phys. Fluids 2, 653 (1959).

19. E. J. OpIK and S. F. SINGER, Phys. Fluids 3, 486 (1960).

20. E. J. OPIK and S. F. SINGer, Phys. Fluids 4, 221 (1961).

21. S. F. Singer, J. Geophys. Res. 65, 2577 (1960).

22. J. W. Chamberlain, Astrophys. J. 131, 47 (1960).

23. J. C. Brandt and J. W. Chamberlain, Phys. Fluids 3, 485 (1960).

24. F. S. Johnson and R. A. Fish, Astrophys. J. 131, 502 (1960).

25. F. S. JoHnson, Astrophys. J. 133, 701 (1961).

26. J. HeRRING and L. KYLE, J. Geophys. Res. 66, 1980 (1961).

27. R. E. AAMODT and K. M. CASE, Phys. Fluids 5, 1019 (1962).

28. J. W. Chamberlain, Planet. Space Sci. 11, 897 (1963).

29. S. ChAPMAN and T. G. Cowling, The Mathematical Theory of Non-Uniform Gases (2nd ed.). Cambridge University Press, Cambridge (1952).

30. H. Grap, Handbuch der Physik, Band XII. Springer-Verlag, Berlin (1958).

31. A. G. WeBsTER, Partial Differential Equations of Mathematical Physics (2nd ed.). G. E. Steckert, Leipzig (1933).

32. H. GoldsteIN, Classical Mechanics. Addison-Wesley, Reading, Mass. (1953). 
33. D. R. BAtes, Proc. Roy. Soc., Lond. A253, 451 (1959).

34. D. R Bates and T. N. L. Patterson, Planet. Space Sci. 5, 257 (1961).

35. D. R. Bates and T. N. L. Patterson, Planet. Space Sci, 9, 599 (1962).

36. M. Krook, P. L. Bhatnager and E. P. Grass, Phys. Rev. 94, 511 (1954).

37. J. L. Delcrorx, Introduction to the Theory of Ionized Gases. Interscience, New York (1960).

38. L. Fox, Numerical Solution of Ordinary and Partial Differential Equations. Addison-Wesley, Reading. Mass. (1962).

Резюме-На основе интегральной формулировки уравнения Больтцмана был сделан анализ соударного перехода между нижними слоями атмосферы и несоударной әкзосферой. В этом исследовании применнется соударная модель, являющаяся комбинацией модели "Лоренц-газ" с соударной моделью "релаксации". Результаты әтого анализа указывают на то, что соударения между молекулами имеют двоякое вовдействие на атмосферу. Во-первых, существует критический слой, сходный с видимой фотосферной поверхностью Солнца, от которого порождается ускользающий из планеты материал. Этот слой обуславливается соударением испытываемым честицами, движущимися на орбитах, несомых планетой в тангенциальном направлении. Во-вторых, соударения сокращают вертикальный поток материала таким же образом, как процессы диффузии, происходящие в нижних слоях атмосферы. 